\title{
二氢噻吩并吡啶-查尔酮衍生物的合成及其体外抗肿瘤活性研究
}

\author{
刘 新 ${ }^{\dagger}$ 许润梅 ${ }^{\dagger}$ 王 淋 ${ }^{\dagger}$ 刘雅雪陈志豪 \\ 秦 巍田玉顺* \\ (延边大学药学院 长白山天然药物研究教育部重点实验室 吉林延吉 133002)
}

\begin{abstract}
摘要 以 2-噻吩乙胺与自制的查尔酮酸进行酰化反应得到酰胺类中间体 $\mathbf{5 a} \sim \mathbf{5 j}$, 经 Bischer-Napieralski 环合反应合成了 10 个未见报道的二氢噻吩并吡啶一查尔酮衍生物 $\mathbf{6 a} \sim \mathbf{6 j}$, 再经去氢反应获得 2 个噻吩并吡啶-查尔酮衍生物 $7 \mathbf{a}$ 和 $7 \mathbf{b}$. 通 过噻唑蓝(MTT)法对 11 种细胞进行体外抗癌活性及安全性测试. 结果表明, 化合物 6a $(p-\mathrm{F}) 、 \mathbf{6 d}(o-\mathrm{Br})$ 和 $\mathbf{6 h}(m-\mathrm{OCH} 3)$ 对 HeLa、SGC-7901 细胞的抗癌活性优于紫杉醇. 当短时间处理 $(<4 \mathrm{~h})$ 时, $\mathbf{6 j}\left(3,4,5-\mathrm{OCH}_{3}\right)$ 在不影响正常细胞 MCF-10A 的情况下对癌细胞 MCF-7 显示强效抗癌效果, 值得进一步研究和开发.
\end{abstract}

关键词 二氢噻吩并吡啶-查尔酮衍生物; 抗癌活性; 选择性; 药物摄取能力

\section{Synthesis and Evaluation in vitro of Dihydrothiophenopyridine- Chalcone Derivatives as Anticancer Activity Agents}

\author{
Liu, Xin ${ }^{\dagger} \quad \mathrm{Xu}$, Runmei $^{\dagger} \quad$ Wang, Lin $^{\dagger} \quad$ Liu, Yaxue $\quad$ Chen, Zhihao \\ Qin, Wei Tian, Yushun* \\ (Key Laboratory of Natural Medicines of the Changbai Mountain, Ministry of Education, College of Pharmacy, \\ Yanbian University, Yanji, Jilin 133002)
}

\begin{abstract}
The amide intermediates $\mathbf{5 a} \sim \mathbf{5 j}$ were prepared by acylation, which used 2-thiopheneethylamine and several self-synthetic chalcone acids as starting materials. Then ten dihydrothiophenopyridine-chalcone derivatives $\mathbf{6 a} \sim \mathbf{6 j}$ which haven't been reported before were synthesized by Bischer-Napieralski cyclization reaction from the intermediates. In addition, two new thiophenopyridine-chalconone derivatives $\mathbf{7 a}$ and $\mathbf{7 b}$ were obtained by further dehydrogenation. The anti-cancer activity and safety in vitro of 11 kinds of cells were evaluated by methyl thiazolyl tetrazolium (MTT) assay. The results indicated that the compounds $\mathbf{6 a}(p-\mathrm{F}), \mathbf{6 d}(o-\mathrm{Br})$ and $\mathbf{6 h}\left(m-\mathrm{OCH}_{3}\right)$ exerted better anticancer activity against HeLa and SGC-7901 cells than taxol. When treating cells for a short time $(<4 \mathrm{~h}), \mathbf{6 j}\left(3,4,5-\left(\mathrm{OCH}_{3}\right)_{3}\right)$ showed strong anticancer activity against MCF-7 cancer cell but displayed little toxicity on normal breast cell MCF-10A. Hence, $\mathbf{6 j}$ deserves further research and exploitation.
\end{abstract}

Keywords dihydrothiophenopyridine-chalcone derivatives; anticancer activity; selectivity; drug uptake capacity

海洋抗癌药物一直是一个热门话题[1]. 从海绵中分 离得到的 ageladine $\mathrm{A}($ 图 1), 为澳化吡咯-咪唑并吡啶类 生物碱，具有抗癌等诸多生物活性，因此在过去几十年 里受到人们的广泛关注 ${ }^{[2-4]}$, 但其天然来源较有限, 且合 成工艺较难, 继之诸多研究者对其衍生物感兴趣. Ma 等 ${ }^{[5]}$ 通过研究其构效关系发现, 原有的氨基咪唑-吡啶 双环结构抗癌效果不明显. 噻吩一吡啶双环结构是一种 典型的咪唑-吡啶的生物电子等排体, 具有抗菌、抗癌、
抗炎、抗糖尿病和抗神经性疾病等广泛的生物活性 ${ }^{[6-10]}$. 肝癌是导致癌症病人死亡最常见原因之一, 其传统化疗 药物为阿霉素等, 均具有较强副作用且疗效并不理 想 ${ }^{[11]} .2010$ 年, Zeng 等 ${ }^{[12]}$ 合成的噻吩并吡啶类化合物对 肝癌细胞的抑制作用约为阿霉素的 23 倍, 因此噻吩-吡 啶双环结构具有重要的研究价值.

查尔酮是黄酮类和异黄酮类化合物生物合成的重 要前体, 主要从绿茶 ${ }^{[13-14]}$ 等天然植物中提取分离得到,

* Corresponding author. E-mail: ystian@ybu.edu.cn

Received January 7, 2021; revised March 15, 2021; published online July 19, 2021.

Project supported by the National Natural Science Foundation of China (No. 81260226) and the College Student Innovation and Entrepreneurship Training Program of Jilin Province in 2020 (No. S202010184016).

国家自然科学基金(No. 81260226)及 2020 年吉林省大学生创新创业训练计划(No. S202010184016)资助项目.

$\dagger$ 共同第一作者(These authors contributed equally to this work). 
其基本骨架为 1,3-二苯基丙烯-1-酮，具有抗肿瘤、抗病 毒、抗炎等广泛的药理作用 ${ }^{[15-22]}$, 近年来对其结构修饰 和生物活性方面的研究极为活跃. 噻吩-吡啶双环结构 与查尔酮结构均为良好的抗癌潜在结构, 但文献中鲜有 噻吩并吡啶一查尔酮衍生物的报道.

本研究中, 利用拼合原理将 ageladine $\mathrm{A}$ 的氨基咪 唑一吡啶双环结构替换成噻吩并吡啶结构, 溴化吡咯部 分替换成查尔酮结构, 设计合成了两个系列、12 个具有 潜在抗癌活性的二氢噻吩并吡啶一查尔酩衍生物(图 1, 化合物 $6 \mathbf{a} \sim 6 \mathbf{j}$ 及 $7 \mathbf{a} \sim 7 \mathbf{b})$, 并探讨其目标产物的抗癌活 性及安全性. 与此同时, 进行了分子对接研究, 以确定 其抗癌活性的关键结构所在.

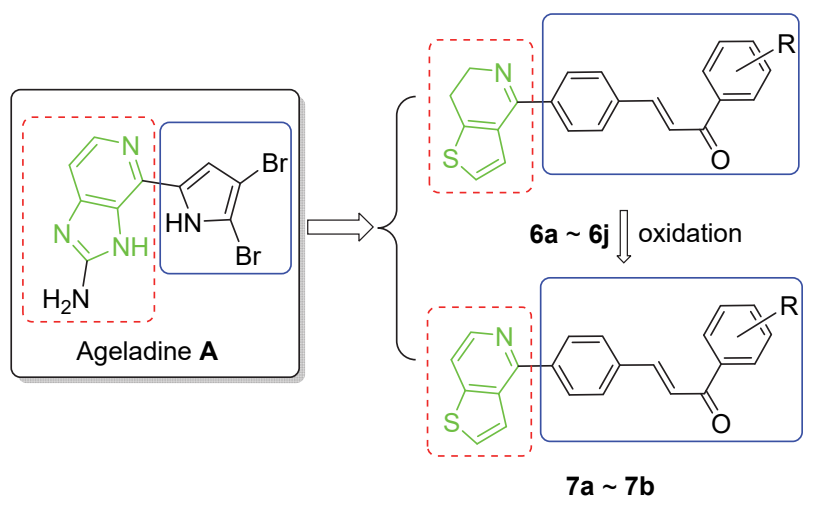

图 1 Ageladine A 及目标化合物 $\mathbf{6 a} \sim \mathbf{6 j}$ 与 $\mathbf{7 a} \sim 7 \mathbf{b}$ 的结构

Figure 1 Structures of ageladine $A$ and the target compounds $\mathbf{6 a} \sim 6 \mathbf{j}$ and $7 \mathbf{a} \sim 7 \mathbf{b}$

\section{1 结果与讨论}

\section{1 目标产物的合成}

目标产物 $\mathbf{6 a} \sim \mathbf{6 j}$ 与 $7 \mathbf{a} \sim 7 \mathrm{~b}$ 的合成路线如 Scheme 1 所示. 按照文献报道的方法, 首先, 在乙醇为溶剂、 $\mathrm{KOH}$ 为催化剂的条件下, 对甲酰基苯甲酸和含不同取 代基的苯乙酮通过 Claisen-Schmidt 缩合反应得到查尔 酮酸 $\mathbf{3 a} \sim 3 \mathbf{j}^{[23]}$. 然后, 查尔酮酸与噻吩乙胺在 1 -乙基(3-二甲基氨基丙基)碳二亚胺盐酸盐(EDC)与 4-二甲氨 基吡啶(DMAP)的催化作用下发生酰化反应得到酰胺类 中间体 $5 \mathbf{a} \sim 5 \mathbf{j}^{[24]}$, 这两步反应产率均较高. 此中间体在 乙腈为溶剂的条件下与 $\mathrm{POCl}_{3}$ 发生 Bischer-Napieralski 环合反应生成二氢噻吩并吡啶类化合物 $\mathbf{6 a} \sim 6 \mathbf{6} \mathbf{j}^{[24]}$, 因色 谱柱上杂质与产物距离近且产物拖尾, 两者分离不佳导 致此步产率较低(12\% 58\%), 其中的部分化合物在 $\mathrm{Pd} / \mathrm{C}$ 作用下去氢生成化合物 $7 \mathbf{a} \sim \mathbf{7 b}^{[25]}$.

\section{2 谱图解析}

所有目标产物经 ${ }^{1} \mathrm{H} \mathrm{NMR} 、{ }^{13} \mathrm{C}$ NMR 和 HRMS 谱图 确证, 以化合物 6b (图 2)为例, 其确证结果如下: $\delta 8.01$ 为苯环上 $\mathrm{C}^{\prime} 、 \mathrm{C} 2$ '位对称氢的吸收峰, $\delta 7.76$ 为苯环上

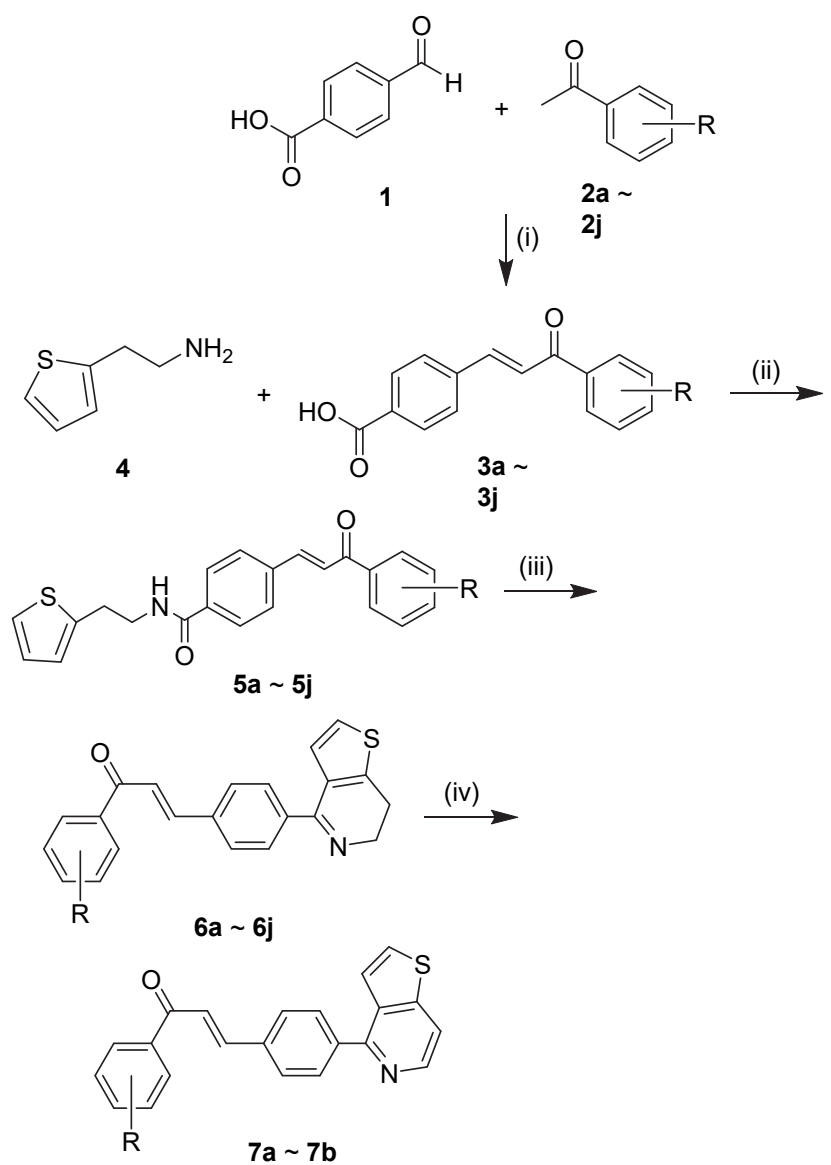

Reagents and conditions: (i) $\mathrm{KOH}, \mathrm{CH}_{2} \mathrm{CH}_{3} \mathrm{OH}$, r.t., overnight $(62 \%$ $100 \%$ ); (ii) EDC, DMAP, $\mathrm{CH}_{2} \mathrm{Cl}_{2}$, r.t., $2 \sim 4 \mathrm{~h}(33 \% \sim 100 \%)$; (iii) $\mathrm{POCl}_{3}$, $\mathrm{CH}_{3} \mathrm{CN}, 60{ }^{\circ} \mathrm{C}$, reflux, $18 \mathrm{~h}(12 \% \sim 58 \%)$; (iv) $\mathrm{Pd} / \mathrm{C}(10 \%)$, reflux, $12 \mathrm{~h}$ (61\% $71 \%)$

图式 1 目标化合物的合成路线

Scheme 1 The synthetic route of the target compounds

C3、C5、C2、C6 位对称氢的吸收峰; $\delta 7.57$ 处的双重峰 为 $\mathrm{C} 7$ 与 $\mathrm{C} 8$ 位 $\mathrm{CH}=\mathrm{CH}$ 上的两个氢的吸收峰, 它们相 互的偶合常数值分别为 $15.7,15.8 \mathrm{~Hz}$, 均大于 $12 \mathrm{~Hz}$, 因 此推测双键构型为 $E$ 型 ${ }^{[26]} ; \delta 7.51$ 为苯环上 $\mathrm{C}^{\prime}$ '、 $\mathrm{C}^{\prime}$ 位 对称氢的吸收峰; $\delta 7.15,7.04$ 分别为噻吩环上 $\mathrm{C} 10 、 \mathrm{C} 11$ 位上氢的吸收峰, $\delta \quad 4.05 \sim 3.97,3.01 \sim 2.96$ 处的三重峰 分别为 $\mathrm{C} 17 、 \mathrm{C} 18$ 位两个 $\mathrm{CH}_{2}$ 上氢的吸收峰. $\delta 188.9$ 处 为羰基 $\mathrm{C} 9$ 位的吸收峰, $\delta 162.0$ 处为二氢吡啶环上 $\mathrm{C} 15$ 位的吸收峰, $\delta 144.6,144.5$ 处为噻吩环和丙烯酮上 C13、 $\mathrm{C} 7$ 位的吸收峰, $\delta 140.9$ 处为连接丙烯酮处苯环 $\mathrm{C} 1$ 位的 吸收峰, $\delta 139.3$ 处为与氯相连苯环 $\mathrm{C}^{\prime}$ 位的吸收峰, $\delta$ 136.4 处为苯环 $\mathrm{C} 4$ 位的吸收峰, $\delta 135.8$ 处为与丙烯碳相 连苯环上 $\mathrm{C} 1$ '位的吸收峰, $\delta 130.3$ 处为噻吩环上 $\mathrm{C} 12$ 位 的吸收峰, $\delta 129.9$ 处为苯环对称碳 C2, C6 位的吸收峰, $\delta$ 129.0, 128.8 处氯相连苯环对称碳 C6'、C2'和 C5'、C3' 位的吸收峰, $\delta 128.4$ 处苯环上对称碳 C3、C5 位的吸收 峰, $\delta 125.6$ 处为噻吩环 $\mathrm{C} 11$ 位的吸收峰, $\delta 122.2$ 处为噻 吩环上 $\mathrm{C} 10$ 位的吸收峰, $\delta 121.9$ 处为丙烯碳双键 $\mathrm{C} 8$ 位 
的吸收峰, $\delta 48.4$ 和 22.2 处为吡啶环上 $\mathrm{C} 17 、 \mathrm{C} 18$ 位的吸 收峰.

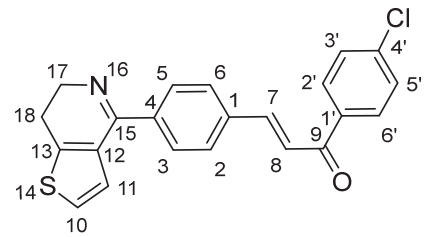

图 2 化合物 $6 \mathrm{~b}$ 的结构

Figure 2 Structure of compound $\mathbf{6 b}$

\section{3 目标化合物的抗癌活性}

所合成的化合物通过噻唑蓝 (MTT) 比色法对 BEL-7402、HepG2、HCT116、A549、MGC-803、 SGC-7901、AGS、HeLa、MCF-7、MCF-10A 与 L-02 等 11 种细胞系进行了体外抗癌活性及选择性评估, 紫 杉醇(taxol)作阳性对照. 考虑到查尔酮酸本身具有一定 的抗癌活性, 故本研究对查尔酮酸 $3 \mathbf{a} \sim \mathbf{3 j}$ 也进行了活 性评估, 并与目标产物进行比较. 结果如表 1 所示, 除 3b $(p-\mathrm{Cl})$ 外, 其他查尔酮酸几乎对每种癌细胞均有一定 的抗癌活性. 化合物 $\mathbf{3 a}(p-\mathrm{F})$ 的抗癌活性普遍优于 $\mathbf{3 c}$ $(p-\mathrm{H})$, 而含溴的化合物对所有癌细胞系均显示出较好 的抗癌活性, 其活性顺序为 $m-\mathrm{Br}>p-\mathrm{Br}>o-\mathrm{Br}$, 其中 $3 \mathbf{e}$ $(m-\mathrm{Br})$ 及 $\mathbf{3 f}(p-\mathrm{Br})$ 对 HCT116、SGC-7901 细胞的抗癌活 性尤为显著, 特别是 3e 及 $3 \mathbf{f}$, 对 SGC-7901 细胞的 $\mathrm{IC}_{50}$ 值分别为(3.2 \pm 0.8$)$ 及 $(3.3 \pm 1.9) \mu \mathrm{mol} / \mathrm{L}$, 远超过 taxol 对 $\mathrm{SGC}-7901$ 细胞的抗癌能力 $\left(\mathrm{IC}_{50}>100 \mu \mathrm{mol} / \mathrm{L}\right)$. 化合物 $\mathbf{3 g}\left(p-\mathrm{CH}_{3}\right) 、 \mathbf{3 h}\left(m-\mathrm{OCH}_{3}\right)$ 及 $\mathbf{3 i}\left(p-\mathrm{OCH}_{3}\right)$ 对每种癌细胞的 抗癌活性相差不大. $3,4,5$ 位有三个甲氧基的化合物 $\mathbf{3 j}$ 对 BEL-7402 的抗癌活性最好, 其 $\mathrm{IC}_{50}$ 值为 $(8.0 \pm 1.4)$ $\mu \mathrm{mol} / \mathrm{L}$.

当这些查尔酮酸与二氢噻吩并吡啶组成新结构后, 除了 $\mathbf{6 b}(p-\mathrm{Cl})$ 及 $\mathbf{6 j}(3,4,5$-三甲氧基)外均对 SGC-7901 细
胞显示出较好的抗癌活性, 其结果如表 2 所示. 多一个 二氢噻吩并吡啶结构的化合物 $6 \mathbf{a}(p-\mathrm{F}) 、 \mathbf{6 c}(\mathrm{H}) 、 \mathbf{6 d}$ $(o-\mathrm{Br})$ 与 $\mathbf{6 h}\left(\mathrm{m}-\mathrm{OCH}_{3}\right)$ 对所有癌细胞的抗癌活性比相应 的查尔酮酸 $(3 \mathbf{a} 、 3 \mathbf{c} 、 3 \mathbf{d}$ 与 $3 \mathrm{~h})$ 显著提高, 但 $\mathbf{6 e}(\mathrm{m}-\mathrm{Br})$ 的抗癌活性普遍比 $3 \mathrm{e}$ 低. 化合物 $3 \mathrm{~b}$ 对 11 种细胞系的 $\mathrm{IC}_{50}$ 值均大于 $100 \mu \mathrm{mol} / \mathrm{L}$, 但具有相同取代基的 $6 \mathbf{b}$ 对 HepG2 与 SGC-7901 两种癌细胞之外的 9 种细胞的抗癌 活性都较强, 说明在对位 $\mathrm{Cl}$ 的存在下，二氢噻吩并吡啶 的引入有利于提高查尔酮的抗癌活性. 将二氢噻吩并吡 啶去氢生成噻吩并吡啶时 $(7 \mathbf{a} 、 7 \mathbf{b})$, 其抗癌活性均明显 下降. 对位有 $\mathrm{F}$ 取代时, $\mathbf{6 a}$ 比查尔酮酸 $\mathbf{3 a}$ 抗癌活性明显 增强, 且 6a 对 SGC-7901 与 HeLa 细胞的抗癌活性显著 优于临床抗癌药物 taxol 的活性. 然而将二氢噻吩并吡 啶去氢成噻吩并吡啶结构 (7a) 时, 除了乳腺癌细胞 MCF-7, 对其它细胞的抗癌活性均明显下降. 值得一提 的是, $7 \mathrm{a}$ 对乳腺正常细胞 MCF-10A 和乳腺癌细胞 MCF-7 的选择性抑制指数 SI [IC 50 (MCF-10A/MCF-7) $]$ 大于 4 , 6a 与 3a 却没有此种选择性抗癌能力. $\mathrm{B}$ 环的不同位置被 $\mathrm{Br}$ 取代的化合物 3d 3f 中, $m-\mathrm{Br}$ 取代物 3e 对所有的癌 细胞及正常细胞的抗增殖作用均强于 $\mathbf{3 d}$ 和 $\mathbf{3 f}$. 但 $\mathbf{6 d} \sim$ 6f 中, $6 \mathbf{d}(o-\mathrm{Br})$ 对所有细胞的抗癌活性最强, 活性顺序 为 $o-\mathrm{Br}>m-\mathrm{Br}>p-\mathrm{Br}$. 虽然 $6 \mathbf{f}(p-\mathrm{Br})$ 的抗癌活性远不如 前两者, 而对部分癌细胞, 如对 $\mathrm{HCT} 116$ 的 $\mathrm{IC}_{50}$ 值为 $(5.2 \pm 2.1) \mu \mathrm{mol} / \mathrm{L}$, 其选择性指数 SI [ $\mathrm{IC}_{50(\mathrm{MCF}-10 \mathrm{~A} / \mathrm{HCT} 116)}$ ] 及 $\left[\mathrm{IC}_{50(\mathrm{~L}-02 / \mathrm{HCT} 116)}\right]$ 分别大于 18 及 7.7. 当 $\mathrm{B}$ 环上有 $p-\mathrm{CH}_{3}$ $(3 \mathrm{~g}$ 和 $6 \mathrm{~g})$ 时, 多一个二氢噻吩并吡啶结构的 $6 \mathrm{~g}$ 对 MGC-803 细胞的活性远大于 $\mathbf{3 g}$, 显示出良好的选择性 抗癌活性, 其对 L-02 的选择性指数为 4.2. 相同位置替 换 $-\mathrm{OCH}_{3}(3 \mathrm{i}$ 和 $6 \mathbf{i})$ 时, $3 \mathbf{i}$ 与 $3 \mathrm{~g}$ 的区别不太明显, 但 $6 \mathrm{~g}$ 对 乳腺癌细胞 $\mathrm{MCF}-7$ 与乳腺正常细胞 $\mathrm{MCF}-10 \mathrm{~A}$ 几乎没有 选择性, 而 $6 \mathbf{i}$ 对这两种细胞的选择性指数大于 4.0 , 说

表 1 目标化合物 $\mathbf{3 a} \sim \mathbf{3} \mathbf{j}$ 的抗癌活性 ${ }^{a}(n \geqslant 3)$

Table 1 Anticancer activity of the compounds $\mathbf{3} \mathbf{a} \sim \mathbf{3} \mathbf{j}^{a}(n \geqslant 3)$

\begin{tabular}{|c|c|c|c|c|c|c|c|c|c|c|c|c|}
\hline \multirow{2}{*}{ Compd. } & \multirow{2}{*}{$\mathrm{R}$} & \multicolumn{11}{|c|}{$\mathrm{IC}_{50} /\left(\mu \mathrm{mol} \cdot \mathrm{L}^{-1}\right)$} \\
\hline & & BEL-7402 & HepG2 & HCT116 & A549 & MGC-803 & SGC-7901 & AGS & MCF-7 & MCF-10A & $\mathrm{HeLa}$ & L-02 \\
\hline $3 a$ & $p$-F & $31.7 \pm 5.4$ & $>100$ & $10.7 \pm 2.2$ & $>100$ & $18.9 \pm 2.1$ & $22.0 \pm 1.3$ & $22.7 \pm 7.8$ & $40.6 \pm 9.2$ & $32.8 \pm 2.8$ & $39.1 \pm 12.1$ & $21.2 \pm 5.7$ \\
\hline $3 \mathbf{b}$ & $p-\mathrm{Cl}$ & $>100$ & $>100$ & $>100$ & $>100$ & $>100$ & $>100$ & $>100$ & $>100$ & $>100$ & $>100$ & $>100$ \\
\hline $3 c$ & $p-\mathrm{H}$ & $14.3 \pm 3.4$ & $>100$ & $21.0 \pm 0.5$ & $>100$ & $>100$ & $>100$ & $32.3 \pm 1.4$ & $24.1 \pm 0.4$ & $47.4 \pm 4.6$ & $35.3 \pm 2.3$ & $27.1 \pm 1.6$ \\
\hline 3d & $o-\mathrm{Br}$ & $25.8 \pm 3.3$ & $94.3 \pm 5.1$ & $8.63 \pm 2.1$ & $>100$ & $15.4 \pm 6.1$ & $26.6 \pm 4.6$ & $22.8 \pm 7.3$ & $45.1 \pm 5.7$ & $37.2 \pm 6.9$ & $69.5 \pm 8.8$ & $23.4 \pm 2.8$ \\
\hline $3 e$ & $m-\mathrm{Br}$ & $5.9 \pm 0.03$ & $11.7 \pm 4.7$ & $3.0 \pm 2.0$ & $20.2 \pm 4.3$ & $5.3 \pm 0.8$ & $3.2 \pm 0.8$ & $5.3 \pm 3.5$ & $13.6 \pm 2.3$ & $8.2 \pm 2.3$ & $23.5 \pm 8.8$ & $5.3 \pm 0.3$ \\
\hline $3 f$ & $p-\mathrm{Br}$ & $10.3 \pm 0.1$ & $35.5 \pm 13.7$ & $3.5 \pm 1.9$ & $57.2 \pm 4.5$ & $9.9 \pm 1.2$ & $3.3 \pm 1.9$ & $12.8 \pm 3.0$ & $17.2 \pm 4.5$ & $16.1 \pm 2.7$ & $15.0 \pm 4.9$ & $11.4 \pm 3.4$ \\
\hline $3 g$ & $p-\mathrm{CH}_{3}$ & $16.0 \pm 2.5$ & $42.0 \pm 3.2$ & $8.0 \pm 1.9$ & $50.8 \pm 3.0$ & $15.1 \pm 1.0$ & $12.5 \pm 1.5$ & $24.7 \pm 7.0$ & $27.4 \pm 3.9$ & $28.9 \pm 5.5$ & $36.3 \pm 7.9$ & $15.6 \pm 1.8$ \\
\hline $3 \mathbf{h}$ & $m-\mathrm{OCH}_{3}$ & $23.6 \pm 2.7$ & $44.5 \pm 15.4$ & $7.2 \pm 0.9$ & $>100$ & $15.6 \pm 0.8$ & $11.7 \pm 1.1$ & $36.7 \pm 10.2$ & $28.0 \pm 3.9$ & $28.8 \pm 7.4$ & $55.4 \pm 8.9$ & $18.8 \pm 2.1$ \\
\hline $3 \mathbf{i}$ & $p-\mathrm{OCH}_{3}$ & $12.3 \pm 3.1$ & $89.6 \pm 0.4$ & $6.4 \pm 3.2$ & $>100$ & $8.5 \pm 0.8$ & $10.7 \pm 2.2$ & $16.9 \pm 1.5$ & $>100$ & $51.1 \pm 5.7$ & $42.6 \pm 8.0$ & $7.5 \pm 1.7$ \\
\hline $3 \mathbf{j}$ & $3,4,5-\left(\mathrm{OCH}_{3}\right)_{3}$ & $8.0 \pm 1.4$ & $>100$ & $>100$ & $>100$ & $11.0 \pm 1.4$ & $>100$ & $11.3 \pm 3.4$ & $21.5 \pm 4.3$ & $38.8 \pm 11.8$ & $39.4 \pm 1.2$ & $5.8 \pm 2.6$ \\
\hline Taxol & & $0.09 \pm 0.02$ & $0.94 \pm 0.03$ & $0.03 \pm 0.01$ & $0.44 \pm 0.02$ & $0.06 \pm 0.01$ & $>100$ & $0.02 \pm 0.01$ & $0.08 \pm 0.01$ & $0.18 \pm 0.02$ & $13.0 \pm 0.4$ & $>100$ \\
\hline
\end{tabular}

${ }^{a}$ The activity of the compound was determined by MTT colorimetry after $48 \mathrm{~h}$ of action, and $\mathrm{IC}_{50}$ was expressed at the concentration required to inhibit $50 \%$ cell proliferation. 
表 2 目标化合物 $\mathbf{6 a} \sim \mathbf{6 j}$ 和 $7 \mathbf{a} \sim 7 \mathbf{b}$ 的抗癌活性 ${ }^{a}(n \geqslant 3)$

Table 2 Anticancer activity of the compounds $\mathbf{6} \mathbf{a} \sim \mathbf{6} \mathbf{j}$ and $7 \mathbf{a} \sim 7 \mathbf{b}^{a}(n \geqslant 3)$

\begin{tabular}{|c|c|c|c|c|c|c|c|c|c|c|c|c|}
\hline \multirow{2}{*}{ Compd. } & \multirow{2}{*}{$\mathrm{R}$} & \multicolumn{11}{|c|}{$\mathrm{IC}_{50} /\left(\mu \mathrm{mol} \cdot \mathrm{L}^{-1}\right)$} \\
\hline & & BEL-7402 & HepG2 & НCT116 & A549 & MGC-803 & SGC-7901 & AGS & MCF-7 & MCF-10A & $\mathrm{HeLa}$ & L-02 \\
\hline $6 a$ & $p-\mathrm{F}$ & $6.0 \pm 0.2$ & $17.8 \pm 2.6$ & $7.6 \pm 0.5$ & $23.6 \pm 4.9$ & $6.6 \pm 1.1$ & $6.0 \pm 0.3$ & $8.3 \pm 2.6$ & $9.0 \pm 3.0$ & $8.5 \pm 2.8$ & $10.5 \pm 2.6$ & $5.6 \pm 1.6$ \\
\hline $6 b$ & $p-\mathrm{Cl}$ & $11.0 \pm 0.4$ & $>100$ & $10.1 \pm 4.1$ & $16.5 \pm 2.6$ & $13.8 \pm 4.0$ & $>100$ & $11.4 \pm 1.4$ & $17.8 \pm 0.7$ & $13.7 \pm 5.0$ & $28.9 \pm 11.3$ & $15.7 \pm 1.1$ \\
\hline $6 c$ & $p-\mathrm{H}$ & $8.7 \pm 1.0$ & $>100$ & $11.8 \pm 2.8$ & $18.3 \pm 9.7$ & $14.3 \pm 2.1$ & $15.1 \pm 6.7$ & $10.4 \pm 7.6$ & $6.40 \pm 1.3$ & $17.9 \pm 4.0$ & $19.4 \pm 2.3$ & $11.3 \pm 2.8$ \\
\hline $6 d$ & $o-\mathrm{Br}$ & $3.8 \pm 4.8$ & $15.6 \pm 0.3$ & $5.1 \pm 0.5$ & $20.2 \pm 3.5$ & $3.6 \pm 1.7$ & $6.7 \pm 0.03$ & $5.7 \pm 2.5$ & $10.2 \pm 1.4$ & $7.6 \pm 1.2$ & $10.9 \pm 5.6$ & $6.9 \pm 1.7$ \\
\hline $6 e$ & $m-\mathrm{Br}$ & $5.9 \pm 2.9$ & $54.5 \pm 31.2$ & $6.5 \pm 2.4$ & $20.7 \pm 1.1$ & $14.7 \pm 5.1$ & $18.3 \pm 1.7$ & $24.3 \pm 5.6$ & $14.1 \pm 5.7$ & $7.4 \pm 2.2$ & $13.9 \pm 5.6$ & $7.2 \pm 3.2$ \\
\hline $6 f$ & $p-\mathrm{Br}$ & $>100$ & $>100$ & $5.2 \pm 2.1$ & $>100$ & $15.7 \pm 0.4$ & $17.3 \pm 6.5$ & $37.0 \pm 12.7$ & $>100$ & $>100$ & $46.1 \pm 1.6$ & $41.1 \pm 2.5$ \\
\hline $6 \mathrm{~g}$ & $p-\mathrm{CH}_{3}$ & $21.8 \pm 3.2$ & $>100$ & $9.3 \pm 1.3$ & $26.5 \pm 5.1$ & $3.4 \pm 2.0$ & $8.2 \pm 0.2$ & $28.7 \pm 8.2$ & $15.1 \pm 3.5$ & $20.3 \pm 11.7$ & $15.2 \pm 4.0$ & $14.9 \pm 6.3$ \\
\hline $6 h$ & $m-\mathrm{OCH}_{3}$ & $5.0 \pm 3.7$ & $19.2 \pm 2.9$ & $6.1 \pm 1.3$ & $15.9 \pm 5.7$ & $5.6 \pm 0.7$ & $9.0 \pm 1.0$ & $5.9 \pm 4.6$ & $12.3 \pm 4.1$ & $10.5 \pm 5.1$ & $7.5 \pm 1.2$ & $6.1 \pm 5.1$ \\
\hline $6 \mathbf{i}$ & $p-\mathrm{OCH}_{3}$ & $24.4 \pm 4.9$ & $>100$ & $24.0 \pm 3.1$ & $23.5 \pm 6.8$ & $8.9 \pm 2.4$ & $11.0 \pm 0.4$ & $17.7 \pm 8.0$ & $24.7 \pm 7.8$ & $>100$ & $21.8 \pm 1.5$ & $11.0 \pm 0.7$ \\
\hline $6 \mathbf{j}$ & $3,4,5-\left(\mathrm{OCH}_{3}\right)_{3}$ & $6.1 \pm 1.3$ & $>100$ & $4.9 \pm 1.7$ & $10.9 \pm 9.2$ & $6.4 \pm 0.5$ & $>100$ & $1.3 \pm 0.1$ & $15.0 \pm 5.0$ & $16.8 \pm 4.3$ & $18.5 \pm 9.7$ & $8.2 \pm 2.0$ \\
\hline $7 a$ & $p-\mathrm{F}$ & $4.5 \pm 0.1$ & $>100$ & $17.3 \pm 3.2$ & $36.2 \pm 2.8$ & $12.3 \pm 0.9$ & $24.1 \pm 6.4$ & $14.5 \pm 3.5$ & $6.5 \pm 1.6$ & $27.6 \pm 3.6$ & $24.6 \pm 4.5$ & $10.7 \pm 5.9$ \\
\hline $7 \mathbf{b}$ & $p-\mathrm{Cl}$ & $62.9 \pm 1.0$ & $>100$ & $21.0 \pm 0.5$ & $>100$ & $>100$ & $75.4 \pm 11.8$ & $48.9 \pm 0.4$ & $56.4 \pm 10.3$ & $>100$ & $>100$ & $32.5 \pm 0.6$ \\
\hline Taxol & & $0.09 \pm 0.02$ & $0.94 \pm 0.03$ & $0.03 \pm 0.01$ & $0.44 \pm 0.02$ & $0.06 \pm 0.01$ & $>100$ & $0.02 \pm 0.01$ & $0.08 \pm 0.01$ & $0.18 \pm 0.02$ & $13.0 \pm 0.4$ & $>100$ \\
\hline
\end{tabular}

${ }^{a}$ The activity of the compound was determined by MTT colorimetry after $48 \mathrm{~h}$ of action, and $\mathrm{IC}_{50}$ was expressed at the concentration required to inhibit $50 \%$ cell proliferation.

明甲基被甲氧基替代后，相应化合物对乳腺癌细胞的选 择性抗癌活性被提高. 在相同的 $\mathrm{OCH}_{3}(\mathbf{6 h}$ 和 $\mathbf{6 i})$ 之间相 比, 间位取代的 $6 \mathbf{h}$ 比对位取代的 $6 \mathbf{i}$ 活性强, 特别是 $\mathbf{6 h}$ 对 HeLa 的抗癌活性(7.5 $\mu \mathrm{mol} / \mathrm{L})$ 超过了紫杉醇 $(13.0$ $\mu \mathrm{mol} / \mathrm{L})$. 当 3,4,5 位有三个 $\mathrm{OCH}_{3}(\mathbf{3} \mathbf{j})$ 时, 其对 $\mathrm{HepG} 2$ 、 HCT116 及 SGC-7901 细胞的抗癌活性明显下降, 但相 同取代基的 $6 \mathbf{j}$ 的抗癌活性普遍强于 $3 \mathbf{j}$, 特别是对 HCT116、AGS 及 A549 的抗癌活性明显增强, 而且也显 示出较强的选择性活性.

抗癌药物一般在体内存留的时间较短, 如果细胞摄 取药物不及时, 则药物无法发挥其抗癌作用. 鉴于 3,4,5- $\left(\mathrm{OCH}_{3}\right)_{3}$ 取代的化合物 $\mathbf{6 j}$ 对 AGS 细胞具有最佳的 抗癌作用, 本研究用含 $\mathbf{6 j}$ 的培养基对 AGS 细胞分别处 理 2、4 或 $6 \mathrm{~h}$ 后换成新鲜培养基继续培养 $48 \mathrm{~h}$, 之后用 MTT 法测定其 $\mathrm{IC}_{50}$ 值. 除了 AGS 细胞外, 对 MCF-7 癌 细胞、L-02 正常细胞与 MCF-10A 正常细胞也进行同样 的操作, 结果如图 3 所示. 结果显示, 处理时间少于 $4 \mathrm{~h}$ 时, $6 \mathbf{j}$ 对 AGS 和 MCF-7 两种癌细胞的抗癌活性依然很 强, 其 $\mathrm{IC}_{50}$ 值均低于 $10 \mu \mathrm{mol} / \mathrm{L}$, 而对 L-02 与 MCF-10A 两种正常细胞, 特别是对 MCF-10A 的抗增殖能力却很 低, 其 $\mathrm{IC}_{50}$ 值超过 $100 \mu \mathrm{mol} / \mathrm{L}$, 乳腺细胞系的选择性指 数 SI [ IC 50 (MCF-10A/MCF-7)] 超过 10 , 意味着短时间 (4h 以内) 用 $6 \mathbf{j}$ 处理时, 对乳腺正常细胞安全的情况下, 对癌细胞 产生很强的抗癌效果.

\section{4 分子对接结果}

Zhang 等 ${ }^{[27]}$ 报道, 微管蛋白的秋水仙碱结合位点中, Ala250、Asn258、Thr179、Lys254 及 Lys352 等 5 个氨 基酸残基是很多抑制剂引起抗癌作用中起关键作用的 氨基酸残基. 经蛋白对接研究发现, 化合物 $6 \mathbf{j}$ 及紫杉醇

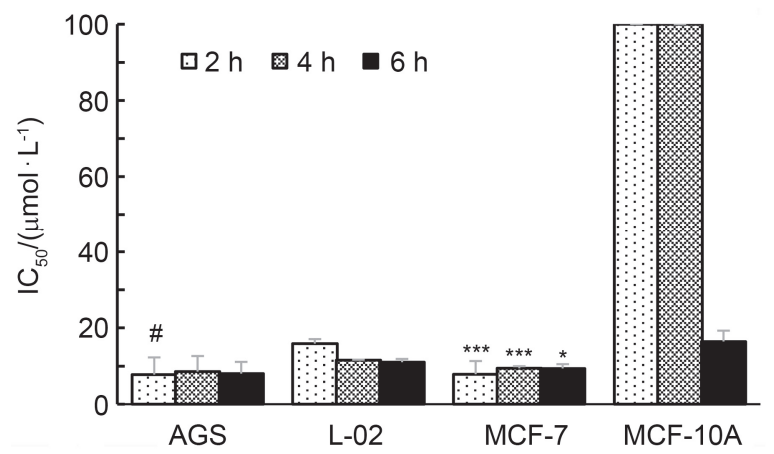

图 3 化合物 $6 \mathbf{j}$ 在不同处理时间下对各种细胞增殖能力的影 响

Figure 3 Effects of compound $\mathbf{6 j}$ on the proliferation of various cells under different treatment time

Values were obtained from three separate experiments and represent the mean \pm SD $(\mathrm{n} \geqslant 3)$. $* P<0.05$ and $* * * P<0.0001$ when the MCF-7 cells and MCF-10A cells groups were compared, and ${ }^{\#} P<0.05$ when the AGS cells and L-02 cells groups were compared.

与微管蛋白形成氢键的情况不同(图 $4 a \sim 4 c$ ). 化合物 $\mathbf{6 j}$ 与微管蛋白的 $\alpha$ 亚基和 $\beta$ 亚基上的氨基酸残基形成的化 学键数量接近, 而且苯环上的两个甲氧基通过 Lys254 与微管蛋白的 $\beta$ 亚基相结合(图 4a), 而紫杉醇则主要停 靠在 $\beta$ 亚基一侧，与 $\alpha$ 亚基上的结合位点只有 Thr179 等三个位点, 大多数结合位点在 $\beta$ 亚基上, 而且通过 Ala250 及 Lys352 等关键的抗癌相关重要氨基酸残基与 微管蛋白 $\beta$ 亚基相结合(图 4b). 两种化合物在 binding pocket 中的叠加结果表明, $\mathbf{6 j}$ 和 taxol 的结合姿态不同, 只有一部分叠加, 是 Leu248 及 Ala354 两个氨基酸残基 与微管蛋白 $\beta$ 亚基相结合的部分有叠加现象(图 4c). 根 据蛋白对接的结果, 可初步判断虽然 $\mathbf{6 j}$ 靶向微管蛋白 的 $\alpha$ 亚基和 $\beta$ 亚基来抑制癌细胞增殖，但只有 Lys254 关键氨基酸残基与靶点结合, 其结合能力远不如紫杉醇 
与微管蛋白的关键氨基酸残基的种类和数目.
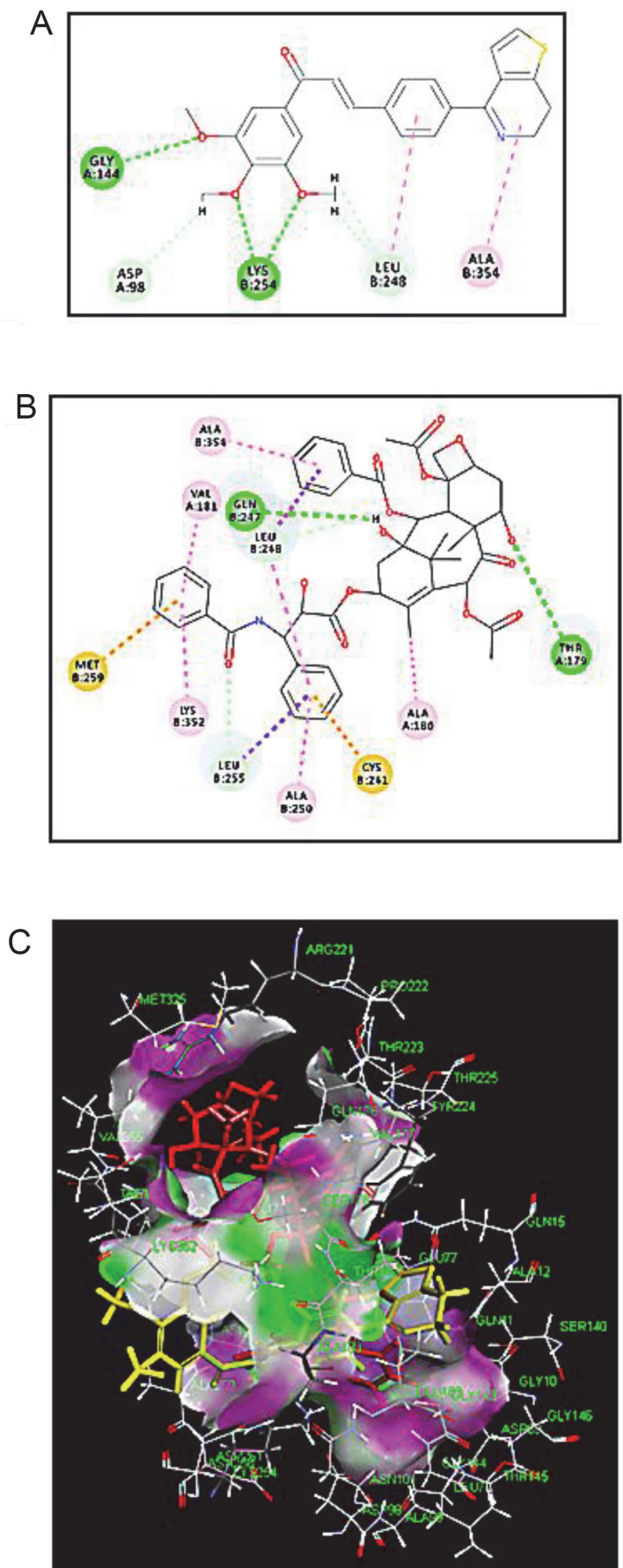

图 4 化合物与微观蛋白(PDB: 1SA0)复合物的分子模拟结果

Figure 4 Molecular modelling of compounds in complex with tubulin (PDB: 1SA0)

Shown is the proposed binding mode and interaction between tubulin and selected compounds. The 2D results of compounds (a) $\mathbf{6 j}$ and (b) taxol. (c) Superposition of $\mathbf{6} \mathbf{j}$ and taxol in the pocket of tubulin in surface representation. The compounds are shown in stick model with carbon atoms in yellow $(\mathbf{6 j})$ and red (taxol).

\section{2 结论}

化合物 $\mathbf{6 a} \sim \mathbf{6 j}$ 对 BEL-7402、HCT116、MGC-803、
SGC-7901、AGS、MCF-7 及 HeLa 具有较好的杀伤作用 $(<10 \mu \mathrm{mol} / \mathrm{L})$ ，其中 6a、6d 对 HeLa, SGC-7901 细胞的 抗癌活性优于紫杉醇. 6 及 7 中较多的化合物，如 6c、6f、 $6 \mathrm{~g} 、 6 \mathrm{i} 、 6 \mathrm{j}$ 与 $7 \mathrm{a}$ 等有很好的选择性抗癌活性. 对乳腺来 源癌细胞 MCF-7 与正常细胞 MCF-10A 来说, 二氢噻吩 并吡啶-查尔酮结构 6 系列中 B 环 $p$-位有供电子基团或 无取代基时，可显示一定的选择性抗癌作用，而吸电性 基团则不具备选择性抗癌活性，但噻吩并吡啶一查尔酮 结构 7 系列的 $\mathrm{B}$ 环 $p$-位有吸电性基团的两个化合物均显 示选择性抗癌活性.

当处理时间较短 $(<4 \mathrm{~h})$ 时, $\mathbf{6 j}$ 对乳腺癌细胞 MCF-7 及乳腺正常细胞 MCF-10A 的选择性抗癌指数超过 10 , 说明用 $6 \mathbf{j}$ 短时间处理可不影响正常细胞的情况下对癌 细胞有强效抗癌效果, 具有作为一种新型抗乳腺癌剂进 一步开发的价值.

蛋白与分子的对接结果表明, $\mathbf{6 j}$ 和 taxol 的结合姿态 不同，两种化合物结构中通过 Leu248 及 Ala354 两个氨 基酸残基与微管蛋白 $\beta$ 亚基相结合的部分有叠加现象. 根据分子与蛋白对接模型的结果，认为 $\mathbf{6 j}$ 通过靶向微 管蛋白的 $\alpha$ 亚基和 $\beta$ 亚基来抑制癌细胞的增殖，但其与 靶点的结合能力不如紫杉醇, 有待进一步完善其结构.

\section{3 实验部分}

\section{1 材料与细胞}

3-(4,5-二甲基噻唑-2-基)-2,5-二苯基- $2 H$-四唑溴化 铵(MTT)购自 Sigma- Aldrich 公司(美国密苏里州圣路易 斯).

BEL-7402 肝癌细胞, HepG2 肝癌细胞, HCT116 肠 癌细胞, A549 肺癌细胞, MGC-803 晚期分化胃癌细胞, SGC-7901 早期分化胃癌细胞, AGS 胃癌细胞, HeLa 宫 颈癌细胞, L-02 正常肝细胞, MCF-7 乳腺癌细胞, MCF-10A 正常乳腺细胞购自 American Type Culture Collection (ATCC, Manassas, VA, USA). RPMI-1640、 DMEM (Dulbecco's modified Eagle's medium)、胎牛血清 (FBS)及 $100 \mathrm{IU} / \mathrm{mL}$ 青霉素和 $100 \mathrm{mg} / \mathrm{mL}$ 链霉素购自 Gibco 公司(Grand Island, NY, USA). 细胞是用 RPMI1640 或 DMEM(含 $1 \%$ 青链霉素和 $10 \%$ FBS), 在 $37{ }^{\circ} \mathrm{C}$ $5 \% \mathrm{CO}_{2}$ 湿润条件下培养.

\section{2 仪器与试剂}

在 AV-300 (Bruker, 瑞士)上获得了 ${ }^{1} \mathrm{H}$ NMR 和 ${ }^{13} \mathrm{C}$ NMR 谱, 并使用 TMS 作为内标准. 化学位移以 $\delta$ 表示, 参考了相应的溶剂峰, 偶合常数 $(J)$ 以赫兹 $(\mathrm{Hz})$ 表示. 高 分辨质谱在 Thermo Scientific Q Exactive 质谱计上测量 (Bruker Dartonik, 德国). 所有试剂均为商用试剂, 均为 分析级试剂. 反应过程用薄层色谱法在硅胶板上进行跟 
踪. 目标化合物的熔点是在开放的玻璃微型管中测定, 未作校正.

\section{3 化合物的合成}

\subsection{1 查尔酮酸 $\mathbf{3 a} \sim 3 \mathbf{j}$ 的合成通法}

将 4 -甲酰苯甲酸(1) $(1.0 \mathrm{mmol})$ 和苯乙䣯 $(2)(1.0$ $\mathrm{mmol})$ 在乙醇 $(15 \mathrm{~mL})$ 中混合, 冰浴下加入氢氧化钾 $(2.0$ $\mathrm{mmol})$, 室温搅拌 $10 \mathrm{~h}$. 加入 $50 \mathrm{~mL}$ 蒸馏水稀释, 滴加稀 盐酸溶液调节 $\mathrm{pH}$ 至 $6 \sim 7$, 固体析出, 抽滤, 用冷乙醇 多次冲洗纯化, 旋干得到产物 $\mathbf{3 a} \sim \mathbf{3} \mathbf{j}$. 其合成按照文献 报道的方法进行, 表征数据与文献一致 ${ }^{[29-32]}$, 其收率及 熔点如表 3 所示.

表 3 化合物 $3 \mathbf{a} \sim 3 \mathbf{j}$ 的收率及熔点 ${ }^{a}$

Table 3 Yields and melting points of compounds $\mathbf{3 a} \sim \mathbf{3} \mathbf{j}$

\begin{tabular}{clcc}
\hline Compd. & \multicolumn{1}{c}{$\mathrm{R}$} & $\mathrm{m} . \mathrm{p} .{ }^{\circ} \mathrm{C}$ & Yield/\% \\
\hline $\mathbf{3 a}$ & $p-\mathrm{H}$ & $212 \sim 213$ & 61.8 \\
$\mathbf{3 b}$ & $p-\mathrm{F}$ & $255 \sim 256$ & 79.7 \\
$\mathbf{3 c}$ & $p-\mathrm{Cl}$ & $259 \sim 260$ & 95.3 \\
$\mathbf{3 d}$ & $o-\mathrm{Br}$ & $232 \sim 233$ & 83.9 \\
$\mathbf{3 e}$ & $m-\mathrm{Br}$ & $206 \sim 207$ & 74.7 \\
$\mathbf{3 f}$ & $p-\mathrm{Br}$ & $246 \sim 247$ & 77.3 \\
$\mathbf{3 g}$ & $p-\mathrm{CH}_{3}$ & $242 \sim 243$ & 81.9 \\
$\mathbf{3 h}$ & $m-\mathrm{OCH}_{3}$ & $101 \sim 102$ & 66.3 \\
$\mathbf{3 i}$ & $p-\mathrm{OCH}_{3}$ & $224 \sim 226$ & 84.6 \\
$\mathbf{3 j}$ & $3,4,5-\left(\mathrm{OCH}_{3}\right)_{3}$ & $223 \sim 224$ & 61.7 \\
\hline
\end{tabular}

${ }^{a}$ Reaction conditions: 4-formylbenzoic acid $(\mathbf{1}, 1.0 \mathrm{mmol})$, acetophenone (2, $1.0 \mathrm{mmol}), \mathrm{KOH}(2.0 \mathrm{mmol}), \mathrm{EtOH}(15 \mathrm{~mL})$, yield after purification.

\subsection{2 酰胺中间体 $\mathbf{5} \mathbf{a} \sim \mathbf{5} \mathbf{j}$ 的合成通法}

按照 Tamayo 等 ${ }^{[24]}$ 的方法, 将 2-噻吩乙胺(4) (1.0 $\mathrm{mmol})$, 查尔酮酸 $\mathbf{3 a} \sim 3 \mathbf{j}(2.0 \mathrm{mmol}), 4,4-$ 二甲氨基吡啶 (1.0 mmol)和 1-乙基-(3-二甲基氨基丙基)碳二亚胺盐酸 盐 $(2.0 \mathrm{mmol})$ 在 $15 \mathrm{~mL}$ 无水二氯甲烷溶液中混合, 反应 装置抽真空并用氩气保护, 室温下搅拌 $4 \mathrm{~h}$, 加入乙酸 乙酯和 $10 \%$ 盐酸溶液萃取, 分离得到的有机层用水和饱 和氯化钠溶液洗涤, 用无水硫酸钠干燥, 减压旋蒸除去 溶剂, 粗产物经硅胶柱层析纯化, 甲醇/二氯甲烷混合液 洗脱得到 $5 \mathbf{a} \sim 5 \mathbf{j}$, 未经表征直接用于下一步反应.

\subsection{3 目标化合物 $\mathbf{6 a} \sim \mathbf{6 j}$ 的合成}

上一步得到的酰胺溶解在乙腈中, 逐滴滴加三氯氧 磷(4 equiv.) 到反应体系中, 在 $60{ }^{\circ} \mathrm{C}$ 搅拌 $24 \mathrm{~h}$, 反应液冷 却至室温, 真空旋蒸除去溶剂, 得到残渣. 将残渣溶解 于二氯甲烷中后用饱和碳酸氢钠水溶液 $(50 \mathrm{~mL})$ 洗涤, 分离有机相, 用饱和氯化钠水溶液洗涤, 无水硫酸钠干 燥, 抽滤, 旋蒸除去溶剂得粗品, 经乙酸乙酯/石油醚 洗脱液及硅胶柱层析纯化得到目标产物 $\mathbf{6 a} \sim \mathbf{6 j}$.

(E)-1-(4-氟苯基)-3-(4-(6,7-二氢噻吩并 [3,2-c]吡啶4-基)苯基)-丙-2-烯-1-酩(6a): 酰胺 5a (0.315 g, 0.917 $\mathrm{mmol})$ 溶解在 $5 \mathrm{~mL}$ 乙腈中, 逐滴滴加三氯氧磷 $(0.483$
$\mathrm{mL}$ )于反应体系中, 具体过程如上所述, 纯化得到橙黄 色固体 6a. 产量: $0.080 \mathrm{~g}$, 收率 27\%. m.p. $113 \sim 114{ }^{\circ} \mathrm{C}$; ${ }^{1} \mathrm{H}$ NMR $\left(300 \mathrm{MHz}, \mathrm{CDCl}_{3}\right) \delta: 8.14 \sim 8.06(\mathrm{~m}, 2 \mathrm{H}, \mathrm{C} 6$ ', C2'-H), 7.85 (d, $J=15.7 \mathrm{~Hz}, 1 \mathrm{H}, \mathrm{C} 7-\mathrm{H}), 7.76$ (q, $J=8.5$ $\mathrm{Hz}, 4 \mathrm{H}, \mathrm{C} 3, \mathrm{C} 5, \mathrm{C} 2, \mathrm{C} 6-\mathrm{H}), 7.58$ (d, $J=15.7 \mathrm{~Hz}, 1 \mathrm{H}$, C8-H), 7.21 (dd, $\left.J=12.0,5.3 \mathrm{~Hz}, 2 \mathrm{H}, \mathrm{C} 5^{\prime}, \mathrm{C}^{\prime}\right), 7.15$ (d, $J=5.2 \mathrm{~Hz}, 1 \mathrm{H}, \mathrm{C} 10-\mathrm{H}), 7.04(\mathrm{~d}, J=5.2 \mathrm{~Hz}, 1 \mathrm{H}, \mathrm{C} 11-\mathrm{H})$, $4.08 \sim 3.92(\mathrm{~m}, 2 \mathrm{H}, \mathrm{C} 17-\mathrm{H}), 3.05 \sim 2.90(\mathrm{~m}, 2 \mathrm{H}, \mathrm{C} 18-\mathrm{H})$; ${ }^{13} \mathrm{C}$ NMR (75 MHz, $\left.\mathrm{CDCl}_{3}\right) \delta$ : $188.6(\mathrm{~s}, \mathrm{C} 9), 167.3(\mathrm{~s}$, C15), $163.9 \sim 162.1$ (s, $J=135.0 \mathrm{~Hz}, \mathrm{C} 4$ ), 144.6 (s, C13), 144.2 (s, C7), 140.8 (s, C1), 136.0 (s, C4), 134.4 (s, C1'), 131.10 (d, $\left.J=9.8 \mathrm{~Hz}, \mathrm{C}^{\prime}, \mathrm{C}^{\prime}\right), 130.3$ (s, C12), 128.8 (s, C2, C6), 128.4 (s, C3, C5), 125.7 (s, C11), 122.2 (s, C10), 121.76 (s, C8), 115.9 (s, C5'), 115.6 (s, C3'), 48.3 (s, C17), 22.2 (s, C18). HRMS(ESI) calcd for $\mathrm{C}_{22} \mathrm{H}_{16} \mathrm{FNOS}[\mathrm{M}+$ $\mathrm{H}]^{+}$362.1009; found 362.1001 .

(E)-1-(4-氯苯基)-3-(4-(6,7-二氢噻吩并 [3,2-c]吡啶4-基)苯基)-丙-2-烯-1-酮(6b): 酰胺 $5 \mathbf{b}$ (2.20 g, 6.78 $\mathrm{mmol}$ ) 溶解在 $5 \mathrm{~mL}$ 乙腈中, 逐滴滴加三氯氧磷 $(3.40$ $\mathrm{mL}$ ) 反应体系中, 具体过程如上所述, 纯化得到橙黄 色固体 6b. 产量: $0.630 \mathrm{~g}$, 收率 30\%. m.p. $145 \sim 148{ }^{\circ} \mathrm{C}$; ${ }^{1} \mathrm{H}$ NMR $\left(300 \mathrm{MHz}, \mathrm{CDCl}_{3}\right) \delta: 8.01(\mathrm{~d}, J=8.6 \mathrm{~Hz}, 2 \mathrm{H}$, C6', C2'-H), 7.88 (d, $J=15.7 \mathrm{~Hz}, 1 \mathrm{H}, \mathrm{C} 7-\mathrm{H}), 7.76$ (d, $J=$ $8.4 \mathrm{~Hz}, 4 \mathrm{H}, \mathrm{C} 3, \mathrm{C} 5, \mathrm{C} 2, \mathrm{C} 6-\mathrm{H}), 7.57$ (d, $J=15.8 \mathrm{~Hz}, 1 \mathrm{H}$, C8-H), 7.51 (d, $\left.J=8.6 \mathrm{~Hz}, 2 \mathrm{H}, \mathrm{C}^{\prime}, \mathrm{C}^{\prime}-\mathrm{H}\right), 7.15$ (d, $J=$ $5.2 \mathrm{~Hz}, 1 \mathrm{H}, \mathrm{C} 10-\mathrm{H}), 7.04(\mathrm{~d}, J=5.2 \mathrm{~Hz}, 1 \mathrm{H}, \mathrm{C} 11-\mathrm{H})$, 4.05-3.97 (m, 2H, C17-H), 3.01 2.96 (m, 2H, C18-H); ${ }^{13} \mathrm{C}$ NMR $\left(75 \mathrm{MHz}, \mathrm{CDCl}_{3}\right) \delta: 188.9$ (s, C9), 162.0 (s, C15), 144.6 (s, C13), 144.5 (s, C16), 140.9 (s, C1), 139.3 (s, C4'), 136.4 (s, C4), 135.8 (s, C1'), 130.3 (s, C12), 129.9 (s, C2, C6), 129.0 (s, C6', C2'), 129.8 (s, C5', C3'), 128.4 (s, C3, C5), 125.6 (s, C11), 122.1 (s, C10), 121.8 (s, C8), 48.4 (s, C17), 22.2 (s, C18). HRMS (ESI) calcd for $\mathrm{C}_{22} \mathrm{H}_{16} \mathrm{ClNOS}[\mathrm{M}+\mathrm{H}]^{+}$378.0713; found 378.0710.

(E)-3-(4-(6, 7-二氢噻吩并 [3,2-c]吡啶-4-基)苯基)-1苯基-丙-2-烯-1-酮 $(\mathbf{6 c})$ : 酰胺 5c $(0.100 \mathrm{~g}, 3.07 \mathrm{mmol})$ 溶 解在 $5 \mathrm{~mL}$ 乙腈中, 逐滴滴加三氯氧磷 $(1.70 \mathrm{~mL})$ 于反应 体系中, 具体过程如上所述, 纯化得到橙黄色固体 $\mathbf{6 c}$. 产量: $0.361 \mathrm{~g}$, 收率 38\%. m.p. $113 \sim 114{ }^{\circ} \mathrm{C} ;{ }^{1} \mathrm{H}$ NMR $\left(300 \mathrm{MHz}, \mathrm{CDCl}_{3}\right) \delta: 8.06\left(\mathrm{~d}, J=7.7 \mathrm{~Hz}, 2 \mathrm{H}, \mathrm{C} 6{ }^{\prime}, \mathrm{C}^{\prime}-\mathrm{H}\right)$, 7.87 (d, $J=15.7 \mathrm{~Hz}, 1 \mathrm{H}, \mathrm{C} 7-\mathrm{H}), 7.78$ (q, $J=8.2 \mathrm{~Hz}, 4 \mathrm{H}$, C3, C5, C2, C6-H), 7.63 (d, J=5.3 Hz, 1H, C8-H), 7.60 (d, $\left.J=3.8 \mathrm{~Hz}, 1 \mathrm{H}, \mathrm{C} 4{ }^{\prime}-\mathrm{H}\right), 7.58 \sim 7.50\left(\mathrm{~m}, 2 \mathrm{H}, \mathrm{C} 5^{\prime}\right.$, C3'-H), 7.15 (d, $J=5.0 \mathrm{~Hz}, 1 \mathrm{H}, \mathrm{C} 10-\mathrm{H}), 7.05$ (d, $J=5.1$ $\mathrm{Hz}, 1 \mathrm{H}, \mathrm{C} 11-\mathrm{H}), 4.11 \sim 3.96$ (m, 2H, C17-H), 3.06 2.93 
(m, 2H, C18-H); ${ }^{13} \mathrm{C}$ NMR (75 MHz, $\left.\mathrm{CDCl}_{3}\right) \delta: 190.3(\mathrm{~s}$, C9), 162.1 (s, C15), 144.6 (s, C13), 144.0 (s, C7), 140.7 (s, C1), 138.1 (s, C1'), 136.1 (s, C4), 132.9 (s, C4'), 130.3 (s, C12), 128.8 (s, C3, C2), 128.6 (s, C6, C5), 128.5 (s, C6', C2'), 128.4 (s, C5', C3'), 125.7 (s, C11), 122.7 (s, C10), 121.8 (s, C8), 48.3 (s, C17), 22.2 (s, C18). HRMS (ESI) calcd for $\mathrm{C}_{22} \mathrm{H}_{17} \mathrm{NOS}[\mathrm{M}+\mathrm{H}]^{+}$344.1103; found 344.1096 .

(E)-1-(2-溴苯基)-3-(4-(6,7-二氢噻吩并 [3,2-c]吡啶4-基)苯基)-丙-2-烯-1-酮(6d): 酰胺 5d (0.401 g, 0.992 $\mathrm{mmol})$ 溶解在 $5 \mathrm{~mL}$ 乙腈中, 逐滴滴加三氯氧磷( 0.558 $\mathrm{mL})$ 于反应体系中, 具体过程如上所述, 纯化得到橙黄 色固体 6d. 产量: 0.090 g. 收率 $23 \%$. m.p. $98 \sim 99{ }^{\circ} \mathrm{C} ;{ }^{1} \mathrm{H}$ NMR $\left(300 \mathrm{MHz}, \mathrm{CDCl}_{3}\right) \delta: 7.75(\mathrm{~d}, J=8.3 \mathrm{~Hz}, 2 \mathrm{H}, \mathrm{C} 2$, C6-H), 7.70 (t, 1H, J=8.9 Hz, 3H, C3, C5, C7-H), 7.50 (d, $\left.J=11.4 \mathrm{~Hz}, 1 \mathrm{H}, \mathrm{C} 5{ }^{\prime}-\mathrm{H}\right), 7.46$ (d, $\left.J=2.5 \mathrm{~Hz}, 1 \mathrm{H}, \mathrm{C} 2^{\prime}-\mathrm{H}\right)$, $7.43(\mathrm{~d}, J=7.5 \mathrm{~Hz}, 1 \mathrm{H}, \mathrm{C} 8-\mathrm{H}), 7.40 \sim 7.34$ (m, 1H, C4'-H), 7.20 (s, 1H, C3'-H), 7.14 (d, $J=4.3 \mathrm{~Hz}, 1 \mathrm{H}, \mathrm{C} 10-\mathrm{H}), 7.02$ $(\mathrm{d}, J=5.2 \mathrm{~Hz}, 1 \mathrm{H}, \mathrm{C} 11-\mathrm{H}), 4.03 \sim 3.96(\mathrm{~m}, 2 \mathrm{H}, \mathrm{C} 17-\mathrm{H})$, $3.00 \sim 2.94(\mathrm{~m}, 2 \mathrm{H}, \mathrm{C} 18-\mathrm{H}) ;{ }^{13} \mathrm{C} \mathrm{NMR}\left(75 \mathrm{MHz}, \mathrm{CDCl}_{3}\right)$ $\delta: 194.5$ (s, C9), 162.0 (s, C15), 145.7 (s, C13), 144.6 (s, C7), 141.0 (s, C1'), 135.6 (s, C1), 133.4 (s, C4), 131.4 (s, C4'), 130.3 (s, C12), 129.2 (s, C5'), 128.8 (s, C2, C6), 128.5 (s, C3, C5), 127.4 (s, C2'), 126.8 (s, C3'), 125.6 (s, C11), 121.8 (s, C10, C8), 119.5 (s, C6'), 48.3 (s, C17), 22.2 (s, C18). HRMS (ESI) calcd for $\mathrm{C}_{22} \mathrm{H}_{16} \mathrm{BrNOS}[\mathrm{M}+\mathrm{H}]^{+}$ 422.0208; found 422.0201 .

(E)-1-(3-溴苯基)-3-(4-(6,7-二氢噻吩并 $[3,2-c]$ 吡啶4-基)苯基)-丙-2-烯-1-酮(6e): 酰胺 5e (0.205 g, 0.507 $\mathrm{mmol})$ 溶解在 $5 \mathrm{~mL}$ 乙腈中, 逐滴滴加三氯氧磷(0.286 $\mathrm{mL})$ 于反应体系中, 具体过程如上所述, 纯化得到橙黄 色固体 6e. 产量: 0.110 g. 收率 58\%. m.p. 139 $140{ }^{\circ} \mathrm{C}$; ${ }^{1} \mathrm{H}$ NMR $\left(300 \mathrm{MHz}, \mathrm{CDCl}_{3}\right) \delta: 8.18$ (s, 1H, C7-H), 7.98 (d, $\left.J=7.8 \mathrm{~Hz}, 1 \mathrm{H}, \mathrm{C} 6{ }^{\prime}-\mathrm{H}\right), 7.88$ (d, $\left.J=15.7 \mathrm{~Hz}, 1 \mathrm{H}, \mathrm{C} 4 '-\mathrm{H}\right)$, 7.77 (dd, $\left.J=14.7,8.2 \mathrm{~Hz}, 5 \mathrm{H}, \mathrm{C} 3, \mathrm{C} 5, \mathrm{C} 2, \mathrm{C} 6, \mathrm{C} 2{ }^{\prime}-\mathrm{H}\right)$, 7.54 (d, $J=15.7 \mathrm{~Hz}, 1 \mathrm{H}, \mathrm{C} 8-\mathrm{H}), 7.42$ (t, $J=7.9 \mathrm{~Hz}, 1 \mathrm{H}$, C3'-H), 7.16 (d, $J=5.2 \mathrm{~Hz}, 1 \mathrm{H}, \mathrm{C} 10-\mathrm{H}), 7.05$ (d, $J=5.2$ $\mathrm{Hz}, 1 \mathrm{H}, \mathrm{C} 11-\mathrm{H}), 4.06 \sim 3.95$ (m, 2H, C17-H), 3.03 2.93 $(\mathrm{m}, 2 \mathrm{H}, \mathrm{C} 18-\mathrm{H}) ;{ }^{13} \mathrm{C} \mathrm{NMR}\left(75 \mathrm{MHz}, \mathrm{CDCl}_{3}\right) \delta: 188.8(\mathrm{~s}$, C9), 162.0 (s, C15), 144.9 (s, C7), 144.6 (s, C13), 141.0 (s, C1'), 139.9 (s, C1), 135.8 (s, C4), 135.7 (s, C4'), 131.5 (s, C3'), 130.3 (s, C12), 130.2 (s, C6'), 128.8 (s, C2, C6), 128.5 (s, C3, C5), 127.0 (s, C2'), 125.7 (s, C11), 123.0 (s, C5'), 122.0 (s, C10), 121.8 (s, C8), 48.4 (s, C17), 22.2 (s, C18). HRMS (ESI) calcd for $\mathrm{C}_{22} \mathrm{H}_{16} \mathrm{BrNOS}[\mathrm{M}+\mathrm{H}]^{+}$
422.0208; found 422.0201.

(E)-1-(4-溴苯基)-3-(4-(6,7-二氢噻吩并 [3,2-c]吡啶4-基)苯基)-丙-2-烯-1-酮(6f)：酰胺 $5 \mathbf{f}$ (0.790 g, 1.95 $\mathrm{mmol})$ 溶解在 $5 \mathrm{~mL}$ 乙腈中, 逐滴滴加三氯氧磷(1.10 $\mathrm{mL})$ 于反应体系中, 具体过程如上所述, 纯化得到浅黄 色固体 6f. 产量: 0.184 g. 收率 $25 \%$. m.p. $154 \sim 156{ }^{\circ} \mathrm{C}$; ${ }^{1} \mathrm{H}$ NMR (300 MHz, $\left.\mathrm{CDCl}_{3}\right) \delta: 7.91(\mathrm{~d}, J=8.5 \mathrm{~Hz}, 2 \mathrm{H}$, C6', C2'-H), 7.85 (d, $J=15.7 \mathrm{~Hz}, 1 \mathrm{H}, \mathrm{C} 7-\mathrm{H}), 7.76$ (d, $J=$ $8.3 \mathrm{~Hz}, 2 \mathrm{H}, \mathrm{C} 2,6-\mathrm{H}), 7.71$ (d, $J=8.4 \mathrm{~Hz}, 2 \mathrm{H}, \mathrm{C} 3, \mathrm{C} 5-\mathrm{H})$, 7.66 (d, $J=8.4 \mathrm{~Hz}, 2 \mathrm{H}, \mathrm{C} 5 ', \mathrm{C} 3 '-\mathrm{H}), 7.53$ (d, $J=15.7 \mathrm{~Hz}$, $1 \mathrm{H}, \mathrm{C} 10-\mathrm{H}), 7.13$ (d, $J=5.2 \mathrm{~Hz}, 1 \mathrm{H}, \mathrm{C} 11-\mathrm{H}), 7.02$ (d, $J=$ $5.2 \mathrm{~Hz}, 1 \mathrm{H}, \mathrm{C} 8-\mathrm{H}), 4.05 \sim 3.94(\mathrm{~m}, 2 \mathrm{H}, \mathrm{C} 17-\mathrm{H}), 3.03$ $2.90(\mathrm{~m}, 2 \mathrm{H}, \mathrm{C} 18-\mathrm{H}) ;{ }^{13} \mathrm{C} \mathrm{NMR}\left(75 \mathrm{MHz}, \mathrm{CDCl}_{3}\right) \delta: 189.2$ (s, C9), 162.0 (s, C15), 144.6 (s, C13, C7), 141.0 (s, C1), 136.8 (s, C1'), 135.8 (s, C4), 131.9 (s, C5', C3'), 130.3 (s, C12), 130.0 (s, C6', C2'), 128.8 (s, C2, C6), 128.4 (s, C3, C5), 128.0 (s, C4'), 125.7 (s, C11), 122.1 (s, C10), 121.8 (s, C8), 48.4 (s, C17), 22.2 (s,18). HRMS (ESI) calcd for $\mathrm{C}_{22} \mathrm{H}_{16} \mathrm{BrNOS}[\mathrm{M}+\mathrm{H}]^{+}$422.0208; found 422.0201.

(E)-3-(4-(6,7-二氢噻吩并 [3,2-c] 吡啶 -4- 基 ) 苯 基)-1-(4-甲苯基)-丙-2-烯-1-酮(6g): 酰胺 $5 \mathrm{~g}$ (0.910 g, $2.68 \mathrm{mmol}$ )溶解在 $5 \mathrm{~mL}$ 乙腈中, 逐滴滴加三氯氧磷 (1.49 mL)于反应体系中, 具体过程如上所述, 纯化得到 浅黄绿色固体 6g. 产量: 0.080 g. 收率 13\%. m.p. 146 $147{ }^{\circ} \mathrm{C} ;{ }^{1} \mathrm{H}$ NMR $\left(300 \mathrm{MHz}, \mathrm{CDCl}_{3}\right) \delta: 7.96(\mathrm{~d}, J=8.1 \mathrm{~Hz}$, 2H, C6', C2'-H), 7.84 (d, J=15.6 Hz, 1H, C7-H), 7.73 (q, $J=8.3 \mathrm{~Hz}, 4 \mathrm{H}, \mathrm{C} 3, \mathrm{C} 5, \mathrm{C} 2, \mathrm{C} 6-\mathrm{H}), 7.60$ (d, $J=15.7 \mathrm{~Hz}$, 1H, C8-H), 7.32 (d, J=7.9 Hz, 2H, C5', C3'-H), 7.13 (d, $J=5.2 \mathrm{~Hz}, 1 \mathrm{H}, \mathrm{C} 10-\mathrm{H}), 7.03$ (d, $J=5.2 \mathrm{~Hz}, 1 \mathrm{H}, \mathrm{C} 11-\mathrm{H})$, $4.07 \sim 3.93(\mathrm{~m}, 2 \mathrm{H}, \mathrm{C} 17-\mathrm{H}), 3.03 \sim 2.89$ (m, 2H, C18-H), $2.45\left(\mathrm{~s}, 3 \mathrm{H}, \mathrm{C}^{\prime}-\mathrm{CH}_{3}\right) ;{ }^{13} \mathrm{C} \mathrm{NMR}\left(75 \mathrm{MHz}, \mathrm{CDCl}_{3}\right) \delta$ : 189.8 (s, C9), 162.1 (s, C15), 144.5 (s, C13), 143.7 (s, C7), 143.6 (s, C4'), 140.7 (s, C1), 136.2 (s, C4), 135.5 (s, C1'), 130.4 (s, C12), 129.3 (s, C5', C3'), 128.7 (s, C6', C2'), 128.7 (s, C2, C6), 128.3 (s, C3, C5), 125.7 (s, C11), 122.7 (s, C10), 121.7 (s, C8), 48.4 (s, C17), 22.2 (s, C18), 21.7 (s, C4'- $\mathrm{CH}_{3}$ ). HRMS (ESI) calcd for $\mathrm{C}_{23} \mathrm{H}_{19} \mathrm{NOS}[\mathrm{M}+\mathrm{H}]^{+}$ 358.1260; found 358.1252.

(E)-3-(4-(6,7-二氢噻吩并 [3,2-c] 吡啶 -4- 基) 苯 基)-1-(3-甲氧基苯基)-丙-2-烯-1-酮(6h): 酰胺 5h (0.553 $\mathrm{g}, 1.56 \mathrm{mmol})$ 溶解在 $5 \mathrm{~mL}$ 乙腈中, 逐滴滴加三氯氧磷 (1.30 mL)于反应体系中, 具体过程如上所述, 纯化得到 橙黄色固体 6h. 产量: 0.080 g. 收率 $12 \%$. m.p. 112 $115{ }^{\circ} \mathrm{C} ;{ }^{1} \mathrm{H}$ NMR $\left(300 \mathrm{MHz}, \mathrm{CDCl}_{3}\right) \delta: 7.85$ (d, $J=15.7$ $\mathrm{Hz}, 1 \mathrm{H}, \mathrm{C} 7-\mathrm{H}), 7.78 \sim 7.73(\mathrm{~m}, 2 \mathrm{H}, \mathrm{C} 2, \mathrm{C} 6-\mathrm{H}), 7.73 \sim 7.68$ 
(m, 2H, C3, C5-H), 7.64 7.61 (m, 1H, C2'-H), 7.61 (dd, $\left.J=3.6,2.6 \mathrm{~Hz}, 1 \mathrm{H}, \mathrm{C} 3^{\prime}-\mathrm{H}\right), 7.57 \sim 7.54(\mathrm{~m}, 1 \mathrm{H}, \mathrm{C} 8-\mathrm{H})$, $7.43\left(\mathrm{t}, J=7.9 \mathrm{~Hz}, 1 \mathrm{H}, \mathrm{C} 6{ }^{\prime}-\mathrm{H}\right), 7.17 \sim 7.13\left(\mathrm{~m}, 1 \mathrm{H}, \mathrm{C} 4{ }^{\prime}-\mathrm{H}\right)$, $7.13 \sim 7.11(\mathrm{~m}, 1 \mathrm{H}, \mathrm{C} 10-\mathrm{H}), 7.02(\mathrm{~d}, J=5.2 \mathrm{~Hz}, 1 \mathrm{H}$, C11-H), $4.03 \sim 3.94$ (m, 2H, C17-H), 3.90 (d, $J=2.4 \mathrm{~Hz}$, $\left.3 \mathrm{H}, \mathrm{C}^{\prime}-\mathrm{OCH}_{3}\right), 2.99 \sim 2.91(\mathrm{~m}, 2 \mathrm{H}, \mathrm{C} 18-\mathrm{H}) ;{ }^{13} \mathrm{C}$ NMR $(75$ $\left.\mathrm{MHz}, \mathrm{CDCl}_{3}\right) \delta: 190.0$ (s, C9), 162.1 (s, C15), 159.9 (s, C3'), 144.6 (s, C13), 144.0 (s, C7), 140.8 (s, C1'), 139.5 (s, C1), 136.1 (s, C4), 130.4 (s, C12), 129.6 (s, C5'), 128.8 (s, C2, C6), 128.3 (s, C3, C5), 125.7 (s, C11), 122.8 (s, C10), 121.7 (s, C8), 121.1 (s, C6'), 119.4 (s, C4'), 112.9 (s, C2'), 55.5 (s, $\left.\mathrm{C}^{\prime}-\mathrm{OCH}_{3}\right), 48.4$ (s, C17), 22.2 (s, C18). HRMS (ESI) calcd for $\mathrm{C}_{23} \mathrm{H}_{19} \mathrm{NO}_{2} \mathrm{~S}[\mathrm{M}+\mathrm{H}]^{+} 374.1209$; found 374.1201.

(E)-3-(4-(6,7-二氢噻吩并 [3,2-c] 吡啶 -4- 基) 苯 基)-1-(4-甲氧基苯基)-丙-2-烯-1-酮(6i): 酰胺 $5 \mathbf{i}$ (1.59 g, $4.46 \mathrm{mmol}$ )溶解在 $5 \mathrm{~mL}$ 乙腈中, 逐滴滴加三氯氧磷 (2.46 mL) 于反应体系中, 具体过程如上所述, 纯化得到 橙黄色固体 6i. 产量: 0.100 g. 收率 20\%. m.p. 134 $136{ }^{\circ} \mathrm{C} ;{ }^{1} \mathrm{H}$ NMR $\left(300 \mathrm{MHz}, \mathrm{CDCl}_{3}\right) \delta: 8.06(\mathrm{~d}, J=8.7 \mathrm{~Hz}$, 2H, C6', C2'-H), 7.83 (d, J=15.9 Hz, 1H, C7-H), 7.74 (dd, $J=14.9,8.2 \mathrm{~Hz}, 4 \mathrm{H}, \mathrm{C} 2, \mathrm{C} 6, \mathrm{C} 3, \mathrm{C} 5-\mathrm{H}), 7.61$ (d, $J=16.0$ $\mathrm{Hz}, 1 \mathrm{H}, \mathrm{C} 8-\mathrm{H}), 7.14$ (d, J=5.0 Hz, 1H, C10-H), 7.04 (d, $J=5.2 \mathrm{~Hz}, 1 \mathrm{H}, \mathrm{C} 11-\mathrm{H}), 7.00(\mathrm{~d}, J=8.5 \mathrm{~Hz}, 2 \mathrm{H}, \mathrm{C} 5$, C3'-H), $4.04 \sim 3.95(\mathrm{~m}, 2 \mathrm{H}, \mathrm{C} 17-\mathrm{H}), 3.90(\mathrm{~s}, 3 \mathrm{H}$, $\left.\mathrm{C} 4^{\prime}-\mathrm{OCH}_{3}\right), 3.03-2.92(\mathrm{~m}, 2 \mathrm{H}, \mathrm{C} 18-\mathrm{H}) ;{ }^{13} \mathrm{C}$ NMR $(75$ $\mathrm{MHz}, \mathrm{CDCl}_{3}$ ) $\delta: 188.5$ (s, C9), 163.5 (s, C15), 162.3 (s, C4'), 144.9 (s, C13), 143.1 (s, C7), 140.2 (s, C1), 136.4 (s, C4), 131.0(s, C12), 130.8 (s, C6', C2'), 130.3 (s, C1'), 128.8 (s, C2, C6), 128.3 (s, C3, C5), 125.8 (s, C11), 122.6 (s, C10), 121.9 (s, C8), 113.9 (s, C5', C3'), 55.5 (s, $\mathrm{C}^{\prime}-\mathrm{OCH}_{3}$ ), 48.1 (s, C17), 22.2 (s, C18). HRMS (ESI) calcd for $\mathrm{C}_{23} \mathrm{H}_{19} \mathrm{NO}_{2} \mathrm{~S}[\mathrm{M}+\mathrm{H}]^{+}$374.1209; found 374.1201 .

(E)-3-(4-(6,7-二氢噻吩并 [3,2-c] 吡啶-4- 基) 苯 基)-1-(3,4,5-甲氧基苯基)-丙-2-烯-1-酮 (6jo): 酰胺 $5 \mathbf{j}$ $(0.530 \mathrm{~g}, 1.28 \mathrm{mmol})$ 溶解在 $5 \mathrm{~mL}$ 乙腈中, 逐滴滴加三氯 氧磷 $(0.720 \mathrm{~mL})$ 于反应体系中, 具体过程如上所述, 纯 化得到橙黄绿色固体 6j. 产量: 0.150 g. 收率 30\%. m.p. $165 \sim 168{ }^{\circ} \mathrm{C} ;{ }^{1} \mathrm{H}$ NMR $\left(300 \mathrm{MHz}, \mathrm{CDCl}_{3}\right) \delta: 7.88(\mathrm{~d}, 1 \mathrm{H}$, C7-H), 7.77 (q, $J=8.3 \mathrm{~Hz}, 4 \mathrm{H}, \mathrm{C} 2$, C6-H, C3, C5-H), 7.56 (d, 1H, J=15.7 Hz, C8-H), 7.32 (s, 2H, C6', C2'-H), 7.16 (d, $J=5.1 \mathrm{~Hz}, 1 \mathrm{H}, \mathrm{C} 10-\mathrm{H}), 7.06(\mathrm{~d}, J=5.2 \mathrm{~Hz}, 1 \mathrm{H}$, C11-H), 4.03 (d, $J=8.1 \mathrm{~Hz}, 2 \mathrm{H}, \mathrm{C} 17-\mathrm{H}$ ), 3.99 (s, 6H, C5', $\left.\mathrm{C}^{\prime}-\mathrm{OCH}_{3}\right), 3.97$ (s, 3H, C4'-OCH 3 ), 3.02 (t, $J=7.9 \mathrm{~Hz}$,
$2 \mathrm{H}, \mathrm{C} 18-\mathrm{H}) ;{ }^{13} \mathrm{C}$ NMR $\left(75 \mathrm{MHz}, \mathrm{CDCl}_{3}\right) \delta: 189.0$ (s, C9), 162.0 (s, C15), 153.2 (s, C5', C3'), 144.6 (s, C13), 143.9 (s, C7), 142.6 (s, C4'), 140.8 (s, C1), 136.1 (s, C4), 133.4 (s, C1'), 130.3 (s, C12), 128.8 (s, C2, C6), 128.3 (s, C3, C5), 125.6 (s, C11), 122.4 (s, C10), 121.7 (s, C8), 106.2 (s, C6', C2'), 61.0 (s, $\left.\mathrm{C} 4^{\prime}-\mathrm{OCH}_{3}\right), 56.4$ (s, C5', $\left.\mathrm{C}^{\prime}-\mathrm{OCH}_{3}\right), 48.4$ (s, C17), 22.2 (s, C18). HRMS (ESI) calcd for $\mathrm{C}_{25} \mathrm{H}_{23} \mathrm{NO}_{4} \mathrm{~S}$ $[\mathrm{M}+\mathrm{H}]^{+}$434.1420; found 434.1411.

\subsection{4 目标化合物 $7 \mathbf{a} \sim 7 \mathbf{b}$ 的合成}

化合物 6a 或 $6 \mathbf{b}$ 的二甲苯溶液中加入 $\mathrm{Pd} / \mathrm{C}(10 \%)$, 反应混合物在氞气保护和 $120{ }^{\circ} \mathrm{C}$ 温度条件下搅拌过夜. 薄层色谱跟踪反应完全, 反应液冷却至室温, 抽滤除去 $\mathrm{Pd} / \mathrm{C}$ (用 $\mathrm{CH}_{2} \mathrm{Cl}_{2}$ 漂洗), 减压去除有机层, 粗产物经乙酸 乙酯/石油醚重结晶纯化得到纯产物 7.

(E)-1-(4-氟苯基)-3-(4-(噻吩并 [3,2-c]吡啶-4-基)苯 基)-丙-2-烯-1-酮(7a): 将 $6 \mathbf{6}(0.100 \mathrm{~g}, 0.293 \mathrm{mmol})$ 溶于 5 $\mathrm{mL}$ 二甲苯溶液中, 加入 $0.1 \mathrm{~g} \mathrm{Pd} / \mathrm{C}(10 \%)$, 反应体系抽 真空并用氩气保护, $120{ }^{\circ} \mathrm{C}$ 反应 $12 \mathrm{~h}$, 具体方法如上所 述, 纯化得到黄色固体 7a. 产量: $0.060 \mathrm{~g}$. 收率 61\%. m.p. $165 \sim 166{ }^{\circ} \mathrm{C} ;{ }^{1} \mathrm{H}$ NMR $\left(300 \mathrm{MHz}, \mathrm{CDCl}_{3}\right) \delta: 8.61(\mathrm{~d}$, $J=5.5 \mathrm{~Hz}, 1 \mathrm{H}, \mathrm{C} 17-\mathrm{H}), 8.12(\mathrm{dd}, J=8.8,5.4 \mathrm{~Hz}, 2 \mathrm{H}, \mathrm{C} 3$, $\mathrm{C} 5-\mathrm{H}), 7.98 \sim 7.93(\mathrm{~m}, 2 \mathrm{H}, \mathrm{C} 18, \mathrm{C} 7-\mathrm{H}), 7.86(\mathrm{~d}, J=11.2$ $\mathrm{Hz}, 1 \mathrm{H}, \mathrm{C} 10-\mathrm{H}), 7.84$ (d, $\left.J=8.1 \mathrm{~Hz}, 2 \mathrm{H}, \mathrm{C} 6{ }^{\prime}, \mathrm{C} 2 '-\mathrm{H}\right)$, $7.69 \sim 7.63(\mathrm{~m}, 2 \mathrm{H}, \mathrm{C} 11, \mathrm{C} 8-\mathrm{H}), 7.58(\mathrm{t}, J=4.5 \mathrm{~Hz}, 2 \mathrm{H}$, C2, C6-H), 7.22 (t, $J=8.6 \mathrm{~Hz}, 2 \mathrm{H}, \mathrm{C} 5$ ', C3'-H); ${ }^{13} \mathrm{C}$ NMR $\left(75 \mathrm{MHz}, \mathrm{CDCl}_{3}\right) \delta: 188.7$ (s, C9), 167.3 163.9 (s, $J=$ $255 \mathrm{~Hz}, \mathrm{C} 4$ ) $), 154.2$ (s, C17), 148.5 (s, C15), 144.3 (s, C13), 142.4 (s, C7), 142.0 (s, C1), 135.2 (s, C12), 134.5 (s, C4), 133.5 (s, C1'), 131.2 (d, C6', C2'), 129.7 (s, C3, C5), 128.7 (s, C2, C6), 127.4 (s, C10), 123.1 (s, C8), 122.1 (s, C18), 116.5 (s, C11), 115.9 (s, C5'), 115.6 (s, C3'). HRMS (ESI) calcd for $\mathrm{C}_{22} \mathrm{H}_{14} \mathrm{FNOS}[\mathrm{M}+\mathrm{H}]^{+}$360.0852; found 360.0846 .

(E)-1-(4-氯苯基)-3-(4-(噻吩并 [3,2-c]吡啶-4-基)苯 基)-丙-2-烯-1-酮(7b): 将 $6 \mathbf{b}(0.100 \mathrm{~g}, 0.308 \mathrm{mmol}$ )溶于 5 $\mathrm{mL}$ 二甲苯溶液中, 加入 $0.1 \mathrm{~g} \mathrm{Pd} / \mathrm{C}(10 \%)$, 反应体系抽 真空并用氩气保护, $120{ }^{\circ} \mathrm{C}$ 反应 $12 \mathrm{~h}$, 具体方法如上所 述, 纯化得到黄色固体 7b. 产量: $0.070 \mathrm{~g}$. 收率 71\%. m.p. $132 \sim 134{ }^{\circ} \mathrm{C} ;{ }^{1} \mathrm{H}$ NMR $\left(300 \mathrm{MHz}, \mathrm{CDCl}_{3}\right) \delta: 8.61(\mathrm{~d}$, $J=5.5 \mathrm{~Hz}, 1 \mathrm{H}, \mathrm{C} 17-\mathrm{H}), 8.03(\mathrm{~d}, J=8.5 \mathrm{~Hz}, 2 \mathrm{H}, \mathrm{C} 3$, C5-H), 7.96 (d, $J=7.9 \mathrm{~Hz}, 2 \mathrm{H}, \mathrm{C} 6$ ', C2'-H), 7.88 (d, $J=$ $9.2 \mathrm{~Hz}, 1 \mathrm{H}, \mathrm{C} 7-\mathrm{H}), 7.83$ (d, J=8.2 Hz, 2H, C11, C18-H), 7.67 (s, 1H, C8-H), 7.63 (s, 1H, C10-H), 7.58 (d, $J=4.2$ Hz, 2H, C2, C6-H), 7.52 (d, J=8.3 Hz, 2H, C5', C3'-H); ${ }^{13} \mathrm{C}$ NMR (75 MHz, $\mathrm{CDCl}_{3}$ ) $\delta: 189.0$ (s, C9), 154.1 (s, 
C17), 148.6 (s, C15), 144.6 (s, C13), 142.2 (s, C7), 141.9 (s, C1), 139.3 (s, C4'), 136.4 (s, C12), 135.2 (s, C1'), 133.6 (s, C4), 129.9 (s, C3, C5), 129.7 (s, C6', C2'), 129.0 (s, C5', C3'), 128.7 (s, C2, C6), 123.1 (s, C10), 122.1 (s, C18, C8), 116.6 (s, C11). HRMS (ESI) calcd for $\mathrm{C}_{22} \mathrm{H}_{14} \mathrm{CINOS}[\mathrm{M}+$ $\mathrm{H}]^{+}$376.0557; found 376.0552 .

\section{4 细胞活性测试}

\subsection{1 细胞增殖抑制率的测试}

将对数生长期的细胞铺在 96 孔板内, 确保细胞密 度在 $30 \% \sim 40 \%$ 左右. 细胞培养过夜后, 分别用四种不 同浓度的化合物(1、10、50 和 $100 \mu \mathrm{mol} / \mathrm{L})$ 处理细胞，紫 杉醇为阳性对照. 细胞孵育 $48 \mathrm{~h}$ 后弃掉原培养基, 加入 $2 \mathrm{mg} / \mathrm{mL}$ MTT 溶液 $150 \mu \mathrm{L}$ 至 96 孔板中, 再孵育 $4 \mathrm{~h}$. 除 去 MTT 溶液, 加入 $150 \mu \mathrm{L}$ 二甲基亚砜(DMSO) (Sigma-Aldrich, St Louis, MO, USA). 摇床摇晃以确保甲瓒 完全被溶解, 测定 $492 \mathrm{~nm}$ 处的吸光度. 至少进行三次独 立实验, 实验结果为表 1 和表 2 所示. 通过吸光度利用 下式计算出细胞增殖抑制率.

抑制率 $(\%)=(1-\mathrm{OD}$ 平均值 $/ O D$ 平均对照 $) \times 100 \%$

$\mathrm{OD}$ 平均值为受试化合物组的平均吸光度值; $\mathrm{OD}$ 平均对照为空 白对照组的平均吸光度值. 化合物活性以抑制 50\%细胞 增殖所需的浓度来表达 $\mathrm{IC}_{50}$.

3.4 .2 暴露于化合物 $\mathbf{6 j}$ 的时间与细胞增殖抑制率相 关关系的测试

按照 3.4.1 的方法, 将贴壁的细胞用 1、10、50 和 $100 \mu \mathrm{mol} / \mathrm{L}$ 的化合物 $\mathbf{6 j}$ 分别处理 2、4 或 $6 \mathrm{~h}$, 弃去原培 养基，用新鲜培养基洗净 2 次，加入含 $10 \% \mathrm{FBS}$ 及 $1 \%$ $\mathrm{P} / \mathrm{S}$ 的新鲜培养基继续培养 $48 \mathrm{~h}$, 然后用 MTT 法测定.

\section{5 分子对接实验}

为了弄清化合物与微管蛋白可能的结合模式, 本研 究中将一种表现良好且有选择性毒性的化合物 $\mathbf{6 j}$ 用于 分子对接研究. 所用的微管蛋白结构来源于蛋白数据库 (PDB code: 1SA0, https://www.rcsb.org/structure/ $1 \mathrm{SA} 0)^{[28]}$. 对接软件为 Discovery Studio 4.0 工作站中 CDOCKER. 最终相互作用模型根据分数确定最终结果, 阳性对照为紫杉醇.

辅助材料(Supporting Information) 目标化合物的核 磁共振氢谱、碳谱和高分辨质谱图. 这些材料可以免费 从本刊网站(http://sioc-journal.cn/)上下载.

\section{References}

[1] Khalifa, S. A. M.; Elias, N.; Farag, M. A.; Chen, L.; Saeed, A.; Hegazy, M. F.; Moustafa, M. S.; Abd, E. A.; Al-Mousawi, S. M.; Musharraf, S. G.; Chang, F. R.; Iwasaki, A.; Suenaga, K.; Alajlani,
M.; Göransson, U.; El-Seedi, H. R. Mar. Drugs 2019, 17, 491.

[2] Fujita, M.; Nakao, Y.; Matsunaga, S.; Seiki, M.; Itoh, Y.; Yamashita, J.; Van Soest, R. W. M.; Fusetani, N. J. Am. Chem. Soc. 2003, 125, 1570 .

[3] Bickmeyer, U.; Thoms, S.; Koch, F.; Mukagatare, L. P.; Silalahi, R.; Sartoris, F. J. PLoS One 2019, 14, e0213771.

[4] Iwata, T.; Otsuka, S.; Tsubokura, K.; Kurbangalieva, A.; Arai, D.; Fukase, K.; Nakao, Y.; Tanaka, K. Chem.-Eur. J. 2016, 22: 14707.

[5] Ma, Y.; Nam, S.; Jove, R.; Yakushijin, K.; Horne, D. Bioorg. Med. Chem. Lett. 2010, 20, 84

[6] Srivastava, B. K.; Solanki, M.; Mishra, B.; Soni, R.; Jayadev, S.; Valani, D.; Jain, M.; Patel, P. R. Bioorg. Med. Chem. Lett. 2007, 17 1924.

[7] Tang, J.; Lackey, K. E.; Dickerson, S. H. Bioorg. Med. Chem. Lett. 2013, 23, 66

[8] Liu, H.; Li, Y.; Wang, X. Y.; Wang, B.; He, H. Y.; Liu, J. Y.; Xiang, M. L.; He, J.; Wu, X. H.; Yang, L. Bioorg. Med. Chem. Lett. 2013, $23,2349$.

[9] Zhou, S. B; Duan, Y. N.; Wang, J.; Zhang, J.; Sun, H. F; Jiang, H. W; Gu, Z. N; Tong, J. H; Li, J. Y; Li, J.; Liu, H. Eur. J. Med. Chem. 2017, 140, 448.

[10] Kühn, F. J. P.; Kuhn, C.; Lückhoff, A. J. Biol. Chem. 2019, 16, 4102 .

[11] Wei, K. R.; Peng, X. B.; Liang, Z. H.; Cen, H. S. China Cancer 2015, 24, 621 (in Chinese) (魏矿荣，彭侠彪，梁智恒，岑惠珊，中国肿瘤, 2015, 24, 621.)

[12] Zeng, X. X.; Zheng, R. L.; Zhou, T.; He, H. Y.; Liu, J. Y.; Zheng, Y.; Tong, A. P.; Xiang, M. L.; Song, X. R.; Yang, S. Y.; Yu, L. T.; Wei, Y. Q.; Zhao, Y. L.; Yang, L. Bioorg. Med. Chem. Lett. 2010, 20, 6282.

[13] Tian, L.; Wu, G. R.; Wang, Y. Northwest Pharm. J. 2007, 22, 218 (in Chinese). (田兰, 吴桂荣, 王岩, 西北药学杂志, 2007, 22, 218.)

[14] Wang, Y. H.; Bai, H.; Dou, D. Q.; Pei, Y. P.; Chen, Y. J.; Li, W.; Kazuo, K.; Xing, S. R; Tamots, N. Northwest Pharm. J. 2004, 19, 253 (in Chinese).

(王英华，白虹，窦德强，裴玉萍，陈英杰，李巍，小池一男，邢 世瑞, 二阶堂保, 西北药学杂志, 2004, 19, 253.)

[15] Jandial, D. D.; Krill, L. S.; Chen, L.; Wu, C.; Ke, Y.; Xie, J.; Hoang, B. H.; Zi, X. Molecules 2017, 22, 462.

[16] Kurt, B. Z.; Kandas, N. O.; Dag, A.; Sonmez, F.; Kucukislamoglu, M. Arab. J. Chem. 2020, 13, 1120.

[17] Sheng, Q. E.; Zhao, W. Q.; Zeng, M.; Xie, Z. B.; Xia, Y. P.; Cui, D. M. Chin. J. Org. Chem. 2019, 39, 703 (in Chinese). (盛琦威, 赵婉秋, 曾明, 谢中袍, 夏雅平, 崔冬梅, 有机化学, 2019, 39, 703.)

[18] Roussaki, M.; Hall, B.; Lima, S. C.; Da, S. A. C.; Wilkinson, S.; Detsi, A. Bioorg. Med. Chem. Lett. 2013, 23, 6436.

[19] Dong, L. R.; Hu, D. Y.; Wu, Z. X.; Chen, J. X.; Song, B. A. Chin. Chem. Lett. 2017, 28, 1566.

[20] Abdullah, M. I.; Mahmood, A.; Madni, M.; Masood, S.; Kashif, M. Bioorg. Chem. 2014, 54, 31.

[21] Anuradha, V.; Pullela, V.; Srinivas, R.; Ranga, R. K.; Manjulatha, M. G.; Purohit, J.; Adhusudana, R. Bioorg. Med. Chem. 2006, 14, 6820 .

[22] Zhao, L.; Jin, H.; Sun, L.; Piao, H.; Quan, Z. Bioorg. Med. Chem. Lett. 2005, 15, 5027.

[23] Zhao, L. J.; Shi, Y.; Liu, W.; Lin, X. M.; Sun, T. M.; Li, Y. L. Chin. J. Med. Chem. 2010, 20, 163 (in Chinese). (赵乐晶, 石玉, 刘巍, 林晓明, 孙铁民，李祎亮，中国药物化学 杂志, 2010, 20, 163.)

[24] Tamayo, N. A.; Bo, Y.; Gore, V.; Ma, V.; Nishimura, N.; Tang, P.; Deng, H.; Klionsky, L.; Lehto, S. G.; Wang, W.; Youngblood, B.; Chen, J.; Correll, T. L.; Bartberger, M. D.; Gavva, N. R.; Norman, M. H. J. Med. Chem. 2012, 55, 1594.

[25] Shumaila, A. M. A.; Puranik, V. G.; Kusurkar, R. S. Tetrahedron 2011, 67, 936

[26] Yan, Y. K.; Xu, Q.; Gao, Y.; Liu, H.; Tang, X. R. Chin. J. Org. 
Chem. 2018, 38, 1763 (in Chinese).

(严映坤, 徐侨, 高扬, 刘辉, 唐孝荣, 有机化学, 2018, 38, 1763.)

[27] Zhang, J.; Li, F.; Li, Y.; Guo, Y.; Wang, J.; Zhang, S. Med. Chem. 2014, 10, 277.

[28] Liu, Y. N.; Wang, J. J.; Ji, Y. T.; Zhao, G. D.; Tang, L. Q.; Zhang, C. M.; Guo, X. L.; Liu, Z. P. J. Med. Chem. 2016, 59, 5341.

[29] Farooq, S.; Ngaini, Z.; Mortadza, N. A. Bull. Korean Chem. Soc. 2020, 41,920

[30] de Souza, A. C. A.; Mori, M.; Sens, L.; Rocha, R. F.; Tizziani, T.; de Souza, L. F. S.; Domeneghini Chiaradia-Delatorre, L.; Botta, M.; Nunes, R. J.; Terenzi, H.; Menegatti, A. C. O. Bioorg. Med. Chem. Lett. 2007, 30, 127350 .

[31] Jakovljevic, K.; Joksovic, M.; Matić, I. Z.; Petrovic, N.; Stanojkovic, T.; Sladić, D.; Vujcic, M.; Janovic, B.; Joksovic, L.; Trifunovic, S.; Marković, V. Med. Chem. Comm. 2018, 9, 1682.

[32] Parveen, Z.; Brunhofer, G.; Jabeen, I.; Erker, T.; Chiba, P.; Ecker, G. F. Bioorg. Med. Chem. Lett. 2014, 22, 2314.

(Fan, Y.) 